\title{
RETRANSLOCACIÓN Y EFICIENCIA EN EL USO DE NUTRIENTES EN BOSQUES DEL CENTRO DE ANTIOQUIA
}

\author{
Reallocation and nutrient use efficiency in Antioquia central forests
}

Palabras clave: eficiencia, uso de nutrientes, retranslocación, caída de hojarasca fina, Quercus humboldtii, Pinus patula, Cupressus lusitanica, Piedras Blancas.

Keywords: nutrient use efficiency, reallocation, fine litter fall, Quercus humboldtii, Pinus patula, Cupressus lusitanica,

Piedras Blancas.

\section{RESUMEN}

Hemos estudiado durante tres años variables relacionadas con nutrientes, tales como la retranslocación, la eficiencia en su uso y la caída de hojarasca fina en un bosque de roble Quercus humboldtii Bonpl. y en plantaciones forestales de pino pátula (Pinus patula) y ciprés (Cupressus lusitanica) de Antioquia, Colombia. Las cantidades de hojarasca foliar regresadas hasta el suelo siguieron la secuencia decreciente roble $\left(5313.3 \mathrm{~kg} \mathrm{ha}^{-1} \mathrm{año}^{-1}\right)>$ pino $\left(4866.5 \mathrm{~kg} \mathrm{ha}^{-1}\right.$ año $\left.^{-1}\right)>$ ciprés $\left(2460.3 \mathrm{~kg} \mathrm{ha}^{-1}\right.$ año$\left.{ }^{1}\right)$. Las especies de coníferas mostraron la mayor eficiencia en el uso de nutrientes para la mayoría de nutrientes estudiados, con excepción del P, el cual alcanzó el máximo absoluto en el bosque de roble, donde además se registró una clara retranslocación de este nutriente, probablemente como resultado de su escasa disponibilidad en estos suelos derivados de cenizas volcánicas. La retranslocación permite la conservación del nutriente reduciendo sus pérdidas por lavado foliar y desde la hojarasca desprendida del dosel. De hecho, encontramos ganancias de $\mathrm{P}$ vía deposición neta -la cual incluye lavado foliar y deposición atmosférica-, lo que indica que la especie absorbe el $\mathrm{P}$ contenido en el agua lluvia desde las hojas antes de que alcance el piso del bosque. La baja eficiencia en el uso de $\mathrm{N}$

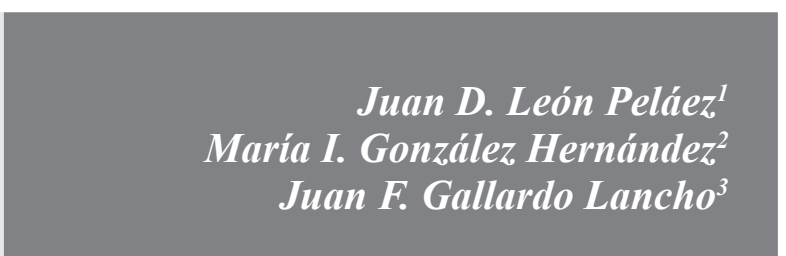

se debe probablemente a su escasa disponibilidad edáfica, dadas sus bajas tasas de mineralización en estos bosques montanos. El K mostró la máxima retranslocación, con valores influenciados por su carácter eminentemente móvil, de acuerdo con los máximos absolutos encontrados para él en la deposición neta. Con excepción del Mg, no se presentó una clara relación entre la retranslocación y la eficiencia en el uso de nutrientes (EUN).

\begin{abstract}
We have studied nutrient related variables such as reallocation, nutrient use efficiency (NUE) and fine litter fall for three years in an oak forest Quercus humboldtii Bonpl. and also in some other forest plantations like pine, Pinus patula, and cypress, Cupressus lusitanica, in Antioquia, Colombia. Leaf litter quantities returned to the soil followed a falling sequence: oak (5313.3 $\mathrm{kg} \mathrm{ha}^{-1}$ year $\left.^{-1}\right)>$ pine $\left(4866.5 \mathrm{~kg} \mathrm{ha}^{-1}\right.$ year $\left.{ }^{-1}\right)>$ cypress $(2460.3 \mathrm{~kg}$ $\mathrm{ha}^{-1}$ year $\left.^{-1}\right)$. The coniferous species showed the highest NUE for the majority of elements that were examined, except for $\mathrm{P}$, which reached its absolute maximum in the oak forest -where a clear reallocation of this nutrient was also recorded-, probably because of its reduced availability in these volcanic
\end{abstract}

1 Universidad Nacional de Colombia, Sede Medellín. Facultad de Ciencias Agropecuarias. A.A. 1779, Medellín, Colombia. Correspondencia: jdleon@unal.edu.co

2 Universidad de Salamanca. Facultad de Ciencias Agrarias y Ambientales. Avd. Filiberto Villalobos, 119. 37007, Salamanca, España.

3 Consejo Superior de Investigaciones Científicas. A.A. 257, Salamanca, España. 
ash-derived soils. Nutrient reallocation allows the conservation of the nutrients by reducing its loss from leaching and litter-fall, thereby closing the nutrient cycle in this native forest. In fact, $\mathrm{P}$ gains from net deposition were found there -this includes foliar leaching and atmospheric deposition-, which indicates that the species absorbs the $\mathrm{P}$ contained in rainfall from the leaves before it reaches the forest ground. N's low-efficiency use was probably due to its low availability in soil, given its low mineralization rates in these montane forests. K showed the highest reallocation values. Such figures are influenced by its clearly mobile character, according to the highest net deposition levels also verified for this element. With the exception of $\mathrm{Mg}$, there was no clear relationship between the reallocation process and NUE.

\section{INTRODUCCIÓN}

El reciclado de nutrientes en los bosques tropicales ha sido calificado como eficiente con respecto al de los bosques templados (Vitousek 1982). Por otra parte, se ha aceptado que la mayoría de las especies usadas en plantaciones forestales son extremadamente conservadoras de los nutrientes del suelo, en relación con su productividad; este es el caso de especies de los géneros Pinus y Eucalyptus (O’Connell \& Sankaran 1997). La retranslocación y la eficiencia en el uso de nutrientes (EUN) son dos aspectos del funcionamiento de los ecosistemas forestales estrechamente ligados entre sí, siendo empleada la primera como indicadora de la EUN en la medida que evalúa la utilización repetida de las mismas unidades de nutriente (Sanz 1992). Dentro de los árboles es un proceso dinámico que varía con la edad y las condiciones de crecimiento y, al parecer, la probabilidad de que algunos nutrientes sean retranslocados está fuertemente asociada con su papel fisiológico. Así, aquellos nutrientes requeridos para la dinámica de algunos procesos $(\mathrm{N}, \mathrm{P}$, $\mathrm{K}, \mathrm{S})$ son móviles y con frecuencia retranslocados. Adicionalmente, la retranslocación representa un mecanismo a través del cual las plantas maximizan la EUN cuando los nutrientes son escasos en el suelo (Del Arco et al. 1991), evitando su pérdida (Vitousek 1982, Chuyong et al. 2000, Hagen-Thorn et al. 2006).
Una aproximación práctica utilizada para la estimación de la EuN (Vitousek 1982) asume que una mayor eficiencia es el resultado de una mayor cantidad de materia orgánica fijada por unidad de nutriente absorbido (Hirose 1975). Esto podría ocurrir bien porque más $\mathrm{C}$ es fijado por unidad de nutriente o bien porque una mayor fracción de los nutrientes es retranslocada desde las partes senescentes de la planta. La retranslocación de nutrientes es considerada como uno de los mecanismos de conservación de nutrientes característicos de gran número de especies tropicales y consiste básicamente en el transporte de nutrientes desde las hojas hacia las ramas, antes de que ocurra la abscisión foliar (Charley \& Richards 1983). Así se aumenta el tiempo de residencia de los nutrientes en los tejidos vegetales, y mejora la EUN a largo plazo (Aerts \& Chapin 2000, Escudero \& Mediavilla 2003, Milla et al. 2005).

La EUN es definida (Vitousek 1982) como la cantidad de $\mathrm{C}$ producida por la planta (como hojarasca y retorno de raíces), más la almacenada en forma permanente (como madera), dividida por la cantidad de nutrientes perdidos (como desfronde, lixiviación en el dosel, o a través del retorno de raíces), más los que permanecen almacenados debido al crecimiento de la vegetación. Sin embargo, es escasa la información disponible de la cantidad de nutrientes almacenados en la madera como consecuencia del crecimiento y la del retorno de raíces. Dadas tales limitaciones, el análisis de la Eun suele considerar aquellos nutrientes aportados al suelo, principalmente vía hojarasca, como N, P y Ca (Cole \& Rapp 1981, Parker 1983), siendo realizado su cálculo (Chapin 1980, Vitousek 1982, 1984; Grubb 1989) como la cantidad de materia seca producida en el desfronde, por unidad de ese nutriente contenido en ella, o, lo que es lo mismo, el inverso de su concentración.

En los bosques montanos tropicales es característica una baja fertilidad edáfica, que usualmente se refleja en bajas concentraciones foliares de nutrientes como N y P (Vitousek 1982, Del Arco et al. 1991, Newbery et al. 1997, Chuyong et al. 2000), lo cual podría conducir a valores altos de EUN. Nosotros hemos estudiado durante tres años 
el proceso de retranslocación de nutrientes en tres bosques montanos maduros de los Andes colombianos (Quercus humboldtii, Pinus patula, Cupressus lusitanica). Para esto la retranslocación ha sido expresada como porcentaje del contenido de cada elemento en la hojarasca foliar con respecto al contenido en las hojas maduras del dosel. Además, para la determinación de la EUN se ha empleado la aproximación del inverso de la concentración del nutriente en la hojarasca foliar. El objetivo de este estudio fue comparar la retranslocación y la EUN de nutrientes foliares entre las tres especies que dominan el bosque natural y las plantaciones forestales bajo las mismas condiciones de suelo y clima. Nuestra hipótesis fue que como especies menos exigentes, las coníferas son menos dependientes del desarrollo de mecanismos de conservación de nutrientes como el de la retranslocación, siendo por lo tanto más eficientes en su uso.

\section{METODOLOGÍA}

\section{ÁREA DE ESTUDIO}

La investigación se realizó entre marzo de 2001 y marzo de 2004 en la cuenca de Piedras Blancas, al sureste de Antioquia, a una distancia de $25 \mathrm{~km}$ de Medellín. Se localiza aproximadamente a 2490 msnm ( $\left.6^{\circ} 18^{\prime \prime} \mathrm{N}, 75^{\circ} 30^{\prime \prime} \mathrm{O}\right)$. Tres parcelas experi- mentales de 0.5 ha fueron seleccionadas en un bosque montano de roble (Quercus humboldtii Bonpl.) $\mathrm{y}$ en plantaciones forestales maduras de pino ( $P i$ nus patula Schltdl. \& Cham.) y ciprés (Cupressus lusitanica Mill., Figura 1). La precipitación media anual es de $1948 \mathrm{~mm}$, tiene una distribución bimodal con máximos en mayo y octubre (León et al. 2008). La temperatura media mensual es de $14.9^{\circ}$ $\mathrm{C}$ con los menores registros en la estación lluviosa. El paisaje comprende colinas de media a baja pendiente cubiertas por capas de cenizas volcánicas sobre un subsuelo de anfibolitas (Tschinkel 1972). Estos suelos derivados de cenizas volcánicas son clasificados como Andepts (Fulvudands y Hapludands, USDA Soil Taxonomy System), tienen altos contenidos de materia orgánica, una baja capacidad de cambio catiónico y altos contenidos de alofanas con gran capacidad de retención de fosfatos (Tabla 1). Los valores de $\mathrm{pH}$ varían entre 4.7 y 5.1 (Rodríguez \& Molina 1990, Jaramillo et al. 2006).

El robledal, dominado por Quercus humboldtii Bonpl. (36\%), contaba con 614 árboles ha ${ }^{-1}$, una estructura vertical compleja, y un sotobosque bien desarrollado. Las plantaciones de pino (Pinus patula Schltdl. \& Cham.) y ciprés (Cupressus lusitanica Mill.) se establecieron hace cerca de 43 años, y no recibieron tratamientos silviculturales dados sus objetivos de conservación (Tabla 2).

Tabla 1. Propiedades de los suelos en los sitios de estudio en la cuenca de Piedras Blancas (Horizonte A: primeros $30 \mathrm{~cm}$ de profundidad).

\begin{tabular}{|c|c|c|c|c|c|c|c|c|c|c|c|c|c|c|}
\hline \multirow{2}{*}{ Bosque } & \multirow{2}{*}{$\mathrm{pH}$} & $S$ & C & $\mathbf{N}$ & \multirow{2}{*}{ C:N } & $\mathrm{Ca}$ & $\mathrm{Mg}$ & K & \multirow{2}{*}{ CIC } & $\mathbf{P}$ & $\mathrm{Fe}$ & Mn & Zn & $\mathrm{Cu}$ \\
\hline & & \multicolumn{3}{|c|}{$(\%)$} & & \multicolumn{3}{|c|}{$\left(\mathrm{c} \mathrm{mol} \mathrm{kg}^{-1}\right)$} & & \multicolumn{5}{|c|}{$\left(\mathrm{mg} \mathrm{kg}^{-1}\right)$} \\
\hline Robledal & 4.7 & 0.05 & 6.70 & 0.32 & 20.51 & 0.16 & 0.18 & 0.13 & 0.47 & 1.10 & 98.78 & 1.36 & 3.16 & 1.00 \\
\hline Pinar & 4.7 & 0.04 & 6.73 & 0.33 & 20.32 & 0.20 & 0.13 & 0.25 & 0.58 & 1.20 & 70.00 & 1.56 & 1.26 & 0.80 \\
\hline Cipresal & 5.1 & 0.06 & 13.80 & 0.60 & 22.87 & 0.10 & 0.13 & 0.25 & 0.48 & 0.70 & 43.20 & 1.70 & 1.04 & 0.40 \\
\hline \multicolumn{15}{|c|}{$\begin{array}{l}\text { Cantidades totales de elementos en el suelo (primeros } 80 \mathrm{~cm} \text { de profundidad). Valores expresados en } \mathrm{Mg} \mathrm{ha}^{-1} \\
\qquad \text { para S, Cy N, y en } \mathrm{kg} \mathrm{ha}^{-1} \text { para Ca, Mg, } \mathrm{K}, \mathrm{P}, \mathrm{Fe}, \mathrm{Mn}, \mathrm{Zn} \text { y Cu}\end{array}$} \\
\hline & & $S$ & C & N & C:N & $\mathrm{Ca}$ & $\mathrm{Mg}$ & $K$ & & $\mathbf{P}$ & $\mathrm{Fe}$ & $M n$ & $\mathrm{Zn}$ & $\mathrm{Cu}$ \\
\hline Robledal & & 2.30 & 232.26 & 10.37 & 22.39 & 132.0 & 30.0 & 0.3 & & 5.0 & 368.0 & 4.0 & 9.0 & 9.0 \\
\hline Pinar & & 1.95 & 188.88 & 8.65 & 21.84 & 120.0 & 20.0 & 1.0 & & 4.0 & 254.0 & 5.0 & 5.0 & 4.0 \\
\hline Cipresal & & 3.08 & 450.34 & 20.84 & 22.09 & 153.0 & 60.0 & 2.0 & & 4.0 & 321.0 & 10.0 & 6.0 & 4.0 \\
\hline
\end{tabular}


Tabla 2. Características estructurales de los bosques estudiados en la cuenca de Piedras Blancas.

\begin{tabular}{|c|c|c|c|c|c|c|}
\hline Bosque & $\begin{array}{l}\text { Edad } \\
\text { (años) }\end{array}$ & $\begin{array}{c}\text { Densidad } \\
\text { rodal* }^{*}\left(\mathrm{ha}^{-1}\right)\end{array}$ & $\begin{array}{c}\text { Altura } \\
\text { media } \\
\text { dosel(m) }\end{array}$ & $\begin{array}{c}\text { DAP } \\
\text { promedio }(\mathrm{cm})\end{array}$ & $\begin{array}{l}\text { Área basal rodal }\left(\mathrm{m}^{2}\right. \\
\left.\qquad \mathrm{ha}^{-1}\right)\end{array}$ & $\begin{array}{c}\text { Biomasa total }{ }^{*}(\mathrm{Mg} \\
\left.\text { ha }^{-1}\right)\end{array}$ \\
\hline Robledal & Maduro & 614 & 10.3 & 15.9 & 17.3 & 166.4 \\
\hline Pinar & 43 & 439 & 19.7 & 23.1 & 41.7 & 328.1 \\
\hline Cipresal & 43 & 615 & 12.5 & 18.2 & 36.6 & 194.8 \\
\hline
\end{tabular}

* Para árboles con DAP $>10 \mathrm{~cm}$.

\section{RECOLECCIÓN DEL MATERIAL FOLIAR}

A comienzos del estudio se seleccionaron 10 árboles de la especie dominante de cada bosque, sobre los cuales se monitoreó el proceso de retranslocación de nutrientes foliares móviles. Cada mes se tomaron muestras de hojas maduras de cada individuo, sin síntomas de ataques de plagas o enfermedades, fotosintéticamente activas, que no correspondieran a estados extremos de edad (renuevos o inmaduras y senescentes). Para la recolección del material se ascendió a los árboles mediante ganchos y sillas de escalada, y se emplearon tijeras podadoras y telescópicas (fotos 1 a 4, anexo fotográfico). El material recolectado en cada rodal fue combinado (previo retiro de ramas y órganos no foliares), y fue seleccionada una muestra para sometimiento a análisis de laboratorio.

Por otra parte, para la recolección de la hojarasca foliar se dispusieron 20 trampas en cada rodal, de
$0.5 \mathrm{~m}^{2}$ de sección circular, localizadas $50 \mathrm{~cm}$ sobre la superficie del terreno y elaboradas en tela de malla fina con el fin de permitir la evacuación efectiva del agua lluvia (fotos 5 y 6 , anexo fotográfico). Para disminuir los efectos del pluviolavado y de la descomposición sobre el material foliar colectado que pudieran alterar su composición química, éste se recogió quincenalmente, siendo únicamente empleado para registro de peso seco el material que no correspondiera al período de análisis de laboratorio, cuya frecuencia fue mensual. Las hojas recogidas de las trampas en el período correspondiente a los análisis de laboratorio fueron combinadas según rodal y fue seleccionada una submuestra para ellos. Los datos de precipitación fueron tomados semanalmente de pluviómetros instalados tanto dentro como fuera de los rodales (fotos 7 y 8 , anexo fotográfico).

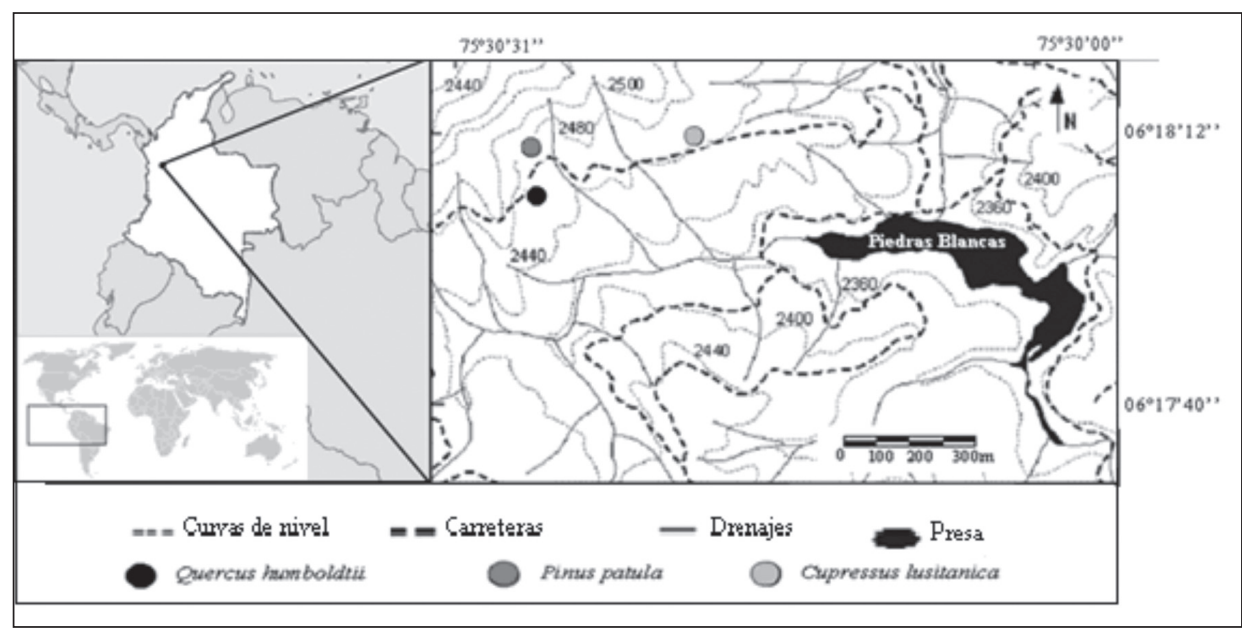

Figura 1. Mapa del área de estudio con las posiciones de los rodales muestreados. Curvas de nivel expresadas en metros. 


\section{DETERMINACIONES QUÍMICAS}

Las hojas maduras y la hojarasca foliar se secaron a $65^{\circ} \mathrm{C}$ (Aussenac et al. 1972) hasta peso constante, se pesaron en balanza analítica y se molieron. Las muestras compuestas para análisis de cationes se llevaron a cenizas a $550^{\circ} \mathrm{C}$ en una mufla, luego se disolvieron en ácido nítrico, se diluyeron y analizaron para $\mathrm{Ca}, \mathrm{Mg}$ y $\mathrm{K}$ por espectrofotometría de absorción atómica. El P total fue determinado colorimétricamente y el $\mathrm{N}$ total por combustión de gases, mediante determinador LECO CNS 2000.

\section{CÁLCULO DE ÍNDICES Y ANÁLISIS ESTADÍSTICO}

\section{Retranslocación de nutrientes}

La retranslocación en las plantas es frecuentemente calculada como la diferencia entre las cantidades de nutrientes en las hojas verdes y en las hojas senescentes (Hagen-Thorn et al. 2006). Este valor, relacionado con la cantidad del elemento en las hojas maduras, permite obtener una aproximación de la cantidad retranslocada del elemento que, multiplicada por 100, la expresa en porcentaje (Del Arco et al. 1991, Ecuación 1).

$$
R=\frac{C h m-C h}{C h m} \times 100
$$

\section{Ecuación 1}

Donde: $\mathrm{Chm}=$ concentración de nutrientes en hojas maduras, $\mathrm{Ch}=$ concentración de nutrientes en la hojarasca. Para determinar la existencia de relaciones entre la retranslocación y la precipitación se practicó un Análisis de Componentes Principales (ACP), en el cual se involucraron, según rodal, los índices de retranslocación obtenidos para $\mathrm{N}, \mathrm{Mg}$, $\mathrm{K}$ y $\mathrm{P}$ y la precipitación incidente. Además fueron obtenidas las cantidades de nutrientes correspondientes al proceso de reabsorción, tomando como punto de partida la diferencia absoluta entre los valores de concentración media de las hojas maduras y de la hojarasca foliar, que multiplicados por la producción de hojarasca foliar de la especie dominante permitieron obtener los flujos expresados en $\mathrm{kg} \mathrm{ha}^{-1} \mathrm{año}^{-1}$.

\section{Eficiencia en el uso de nutrientes}

Se empleó el índice de eficiencia de Vitousek (IEV, 1982), el cual define la eficiencia con la que es utilizado un nutriente como la cantidad de materia seca producida en el desfronde, por unidad de ese nutriente contenida en él, es decir, el inverso de la concentración (Ecuación 2).

$$
I E V=\left(\frac{P s h}{P s e h}\right)
$$

Ecuación 2

Donde: Psh $=$ peso seco de la hojarasca foliar, $\mathrm{y}$ Pseh $=$ peso seco del nutriente en la hojarasca foliar. El empleo de esta aproximación implica (Smith et al. 1998) asumir que los rodales se encuentran en un estado cercano al equilibrio, que la productividad primaria neta es igual a la caída de hojarasca y que los nutrientes perdidos en la hojarasca son equivalentes a aquellos tomados por los bosques. La EUN, así definida, fu e calculada para N, K, P, Ca y Mg.

\section{RESULTADOS}

\section{CONCENTRACIONES FOLIARES}

Las concentraciones de nutrientes en las hojas maduras fueron relativamente semejantes para las tres especies (Tabla 3), presentándose las diferencias más notorias para $\mathrm{N}$ y Ca en ciprés. El nutriente mayoritario fue $\mathrm{N}$ tanto en las hojas maduras como en las hojas muertas, con máximos absolutos para roble. En términos generales las menores concentraciones tanto en hojas verdes como muertas se registraron en ciprés, con excepción de la marcada diferencia de $\mathrm{Ca}$, para el cual se presentaron allí concentraciones foliares de más del doble de magnitud que en las otras dos especies. La abundancia de nutrientes en las hojas maduras siguió la secuencia decreciente $\mathrm{N}>\mathrm{K}>\mathrm{Ca}>\mathrm{Mg}>\mathrm{P}$, siendo la excepción ciprés, que mostró a $\mathrm{Ca}$ en segundo lugar de abundancia, y de casi igual magnitud que N. La secuencia de abundancia en la hojarasca foliar siguió el modelo $\mathrm{N}>\mathrm{Mg}>\mathrm{K}>\mathrm{Ca}>\mathrm{P}$, siendo nuevamente la excepción $\mathrm{Ca}$ en ciprés. 
Tabla 3. Valores medios anuales de concentración de nutrientes en las hojas maduras (Hv) y en las hojas muertas (Hm) de las tres especies (entre paréntesis error). Valores expresados en $\mathrm{mg} \mathrm{g}^{-1}$.

\begin{tabular}{|c|c|c|c|c|c|c|}
\hline Especie & Tipo de hojas & $\mathbf{N}$ & $\mathrm{Ca}$ & $\mathrm{Mg}$ & $K$ & $\mathbf{P}$ \\
\hline \multirow{4}{*}{ Roble } & \multirow{2}{*}{$\mathrm{Hv}$} & $16.0 \mathrm{a}$ & $2.7 \mathrm{a}$ & $1.1 \mathrm{a}$ & $3.3 \mathrm{a}$ & $0.5 \mathrm{a}$ \\
\hline & & $(0.5)$ & $(0.4)$ & (0.1) & $(0.7)$ & $(0.1)$ \\
\hline & \multirow{2}{*}{$\mathrm{Hm}$} & $9.6 \mathrm{a}$ & $0.4 \mathrm{a}$ & $0.9 a$ & $0.8 \mathrm{a}$ & $0.3 \mathrm{a}$ \\
\hline & & $(0.6)$ & $(0.0)$ & (0.1) & (0.3) & $(0.0)$ \\
\hline \multirow{4}{*}{ Pino } & \multirow{2}{*}{$\mathrm{Hv}$} & $15.4 \mathrm{a}$ & $2.8 \mathrm{a}$ & $1.1 \mathrm{a}$ & $3.6 \mathrm{a}$ & $0.5 \mathrm{a}$ \\
\hline & & (1.1) & $(0.6)$ & $(0.1)$ & $(0.5)$ & (0.1) \\
\hline & \multirow{2}{*}{$\mathrm{Hm}$} & $9.2 \mathrm{a}$ & $0.4 \mathrm{a}$ & $1.0 \mathrm{a}$ & $0.6 \mathrm{~b}$ & $0.3 \mathrm{~b}$ \\
\hline & & $(0.7)$ & $(0.1)$ & (0.1) & (0.6) & $(0.0)$ \\
\hline \multirow{4}{*}{ Ciprés } & \multirow{2}{*}{$\mathrm{Hv}$} & $7.6 \mathrm{~b}$ & $7.4 \mathrm{~b}$ & $0.9 \mathrm{~b}$ & $2.7 \mathrm{a}$ & $0.4 \mathrm{ab}$ \\
\hline & & $(0.6)$ & (1.1) & $(0.1)$ & (0.4) & $(0.0)$ \\
\hline & \multirow{2}{*}{$\mathrm{Hm}$} & $5.1 \mathrm{~b}$ & $1.2 \mathrm{~b}$ & $0.6 \mathrm{~b}$ & $0.7 \mathrm{~b}$ & $0.3 \mathrm{~b}$ \\
\hline & & $(1.2)$ & $(0.2)$ & $(0.0)$ & $(0.2)$ & $(0.0)$ \\
\hline
\end{tabular}

Valores correspondientes al mismo tipo de hojas ( $\mathrm{Hv}$ y $\mathrm{Hm}$ ) con letras diferentes son estadísticamente diferentes (prueba de Tukey, $\mathrm{P}<0.05$ ).

\section{RETRANSLOCACIÓN DE NUTRIENTES}

En la Tabla 4 se muestran los valores medios anuales de retranslocación para las tres especies. Aún cuando ha sido ampliamente reportado el carácter poco móvil del Ca (Vera et al. 1999), las diferencias entre sus concentraciones en hojas verdes y hojas maduras fueron representativas (Tabla 3 ).

La menor retranslocación media anual de $\mathrm{N}$ se encontró para ciprés $\left(26.6 \%\right.$ y $\left.5.2 \mathrm{~kg} \mathrm{ha}^{-1}\right)$. Los valores medios de retranslocación de $\mathrm{P}$ fueron similares para pino y ciprés $(18.8 \%$ y $12.4 \%$, respectivamente). A partir del $\mathrm{P}$ extraíble del suelo $\left(\mathrm{H}_{2} \mathrm{PO}_{4}^{-}\right)$ y dadas sus condiciones de acidez, no se observó una clara relación entre estos dos parámetros y la retranslocación de P. Para ciprés la retranslocación de $\mathrm{P}$ fue nuevamente la menor, conforme ocurrió con N. La retranslocación de K fue muy similar entre las coníferas y muy superior a la de roble. Para roble y pino, los valores medios de retranslocación de $\mathrm{Mg}$ fueron los más bajos de los nutrientes estu- diados y, además, muy similares entre sí $(16.0 \%$ y $15.1 \%$ respectivamente). Por el contrario, para ciprés el porcentaje de reabsorción de $\mathrm{Mg}$ fue dos veces superior al encontrado para esas especies $(32.3 \%)$.

\section{DINAMICA DE LA RETRANSLOCACIÓN DE NUTRIENTES}

El primer componente del ACP (Tabla 5) explicó más del $50 \%$ de la variación de los datos para las tres especies y fue superior al $72 \%$ cuando se incluyó el segundo componente, teniendo los dos valores propios mayores a 1.0. La cercanía de los vectores al círculo unitario indicó una buena representación de las variables consideradas. Se verificó una asociación negativa entre la retranslocación (eje 1) y la precipitación para roble y pino (Figura 2); según el primer componente de ambas (Tabla 6) todos los pesos de las variables de retranslocación fueron negativos, en contraposición a la dirección positiva de los pesos de la precipitación incidente.

Tabla 4. Valores medios anuales de retranslocación para las tres especies (\%, entre paréntesis error), y flujo medio anual de nutrientes por retranslocación $\left(\mathrm{kg} \mathrm{ha}^{-1}\right)$ en los tres bosques.

\begin{tabular}{cccccc}
\hline Bosque & Retranslocación & $\mathbf{N}$ & $\mathbf{M g}$ & $\mathbf{K}$ & $\mathbf{P}$ \\
\hline \multirow{2}{*}{ Robledal } & Relativa (\%) & $35.6 \mathrm{a}(5.0)$ & $16.0 \mathrm{a}(8.0)$ & $39.1 \mathrm{a}(30.0)$ & $31.4 \mathrm{a}(12.0)$ \\
& Flujo $\left(\mathrm{kg} \mathrm{ha}^{-1}\right)$ & 17.1 & 0.6 & 5.9 & 0.5 \\
\hline
\end{tabular}




\begin{tabular}{cccccc}
\hline Bosque & Retranslocación & N & Mg & K & P \\
\hline \multirow{2}{*}{ Pinar } & Relativa (\%) & $37.0 \mathrm{a}(8.0)$ & $15.1 \mathrm{a}(13.0)$ & $77.4 \mathrm{a}(4.0)$ & $18.8 \mathrm{a}(12.0)$ \\
& Flujo $\left(\mathrm{kg} \mathrm{ha}^{-1}\right)$ & 28.5 & 0.8 & 13.4 & 0.6 \\
\hline Cipresal & Relativa (\%) & $26.6 \mathrm{a}(5.0)$ & $32.3 \mathrm{~b}(7.0)$ & $65.5 \mathrm{a}(11.0)$ & $12.4 \mathrm{a}(11.0)$ \\
& Flujo $\left(\mathrm{kg} \mathrm{ha}^{-1}\right)$ & 5.2 & 0.8 & 4.7 & 0.2 \\
\hline
\end{tabular}

Valores con letras diferentes son estadísticamente diferentes (Prueba de Tukey, $\mathrm{P}<0.05$ ).

Tabla 5. Valores propios del ACP por especie

\begin{tabular}{ccccc}
\hline Especie & Componente & Valor propio & Porcentaje & $\begin{array}{c}\text { Porcentaje } \\
\text { acumulado }\end{array}$ \\
\hline \multirow{3}{*}{ Roble } & 1 & 28.23 & 56.5 & 56.5 \\
& 2 & 11.12 & 22.2 & 78.7 \\
& 3 & 0.58 & 11.7 & 90.4 \\
& 4 & 0.35 & 7.0 & 97.4 \\
Pino & 5 & 0.13 & 2.6 & 100.0 \\
& 1 & 25.57 & 51.1 & 51.1 \\
& 2 & 10.53 & 21.1 & 72.2 \\
& 3 & 0.57 & 11.5 & 83.7 \\
& 4 & 0.46 & 9.1 & 92.8 \\
& 5 & 0.36 & 7.2 & 100.0 \\
\hline \multirow{3}{*}{ Ciprés } & 1 & 25.37 & 50.8 & 50.8 \\
& 2 & 11.28 & 22.6 & 73.4 \\
& 3 & 0.81 & 16.1 & 89.5 \\
& 4 & 0.36 & 7.3 & 96.8 \\
& 5 & 0.17 & 3.2 & 100.0 \\
\hline
\end{tabular}

Aunque podría esperarse que la mayor afectación de la lluvia sobre el cálculo de la retranslocación fuera para $\mathrm{K}$, su valor para roble fue el más bajo entre todos los nutrientes (-0.65) en el primer componente (Tabla 6); sin embargo, cobró mayor importancia en el segundo componente, donde alcanzó el máximo absoluto (-0.42). Una situación similar ocurrió con este nutriente para pino, indicando el comportamiento diferencial del ciprés respecto a las otras dos especies, originado posiblemente en un carácter basófilo más acentuado, y sus mayores exigencias en la calidad del sitio, constituyéndose así como factores limitantes los edáficos antes que los climáticos.

En el primer componente de ciprés (Tabla 6) todos los pesos de las variables presentaron signos positivos, sin establecerse contraste entre ellas; no obstante en el segundo apareció de nuevo el patrón inverso entre la precipitación y la retranslocación de N, Mg y K. Los mejores ajustes de los modelos de regresión para la retranslocación de $\mathrm{P}(\mathrm{ReP})$ en función de la retranslocación de $\mathrm{N}(\mathrm{ReN})$ se encontraron en roble (Tabla 7), en donde se observó la mayor retranslocación de $\mathrm{P}$. Todos los coeficientes de correlación lineal para la retranslocación de ambos nutrientes (Tabla 8) fueron significativos $(\mathrm{P}<$ $0.05)$ para las tres especies.

\section{EFICIENCIA EN EL USO DE NUTRIENTES}

Ciprés mostró la mayor eficiencia en el uso de N, seguida de pino y roble (Tabla 9). Al representar la EUN (razón peso seco: nutriente) frente al contenido de nutriente en la hojarasca fina (Figuras 3 a 5), se observó para $\mathrm{N}$ en roble y pino un modelo 

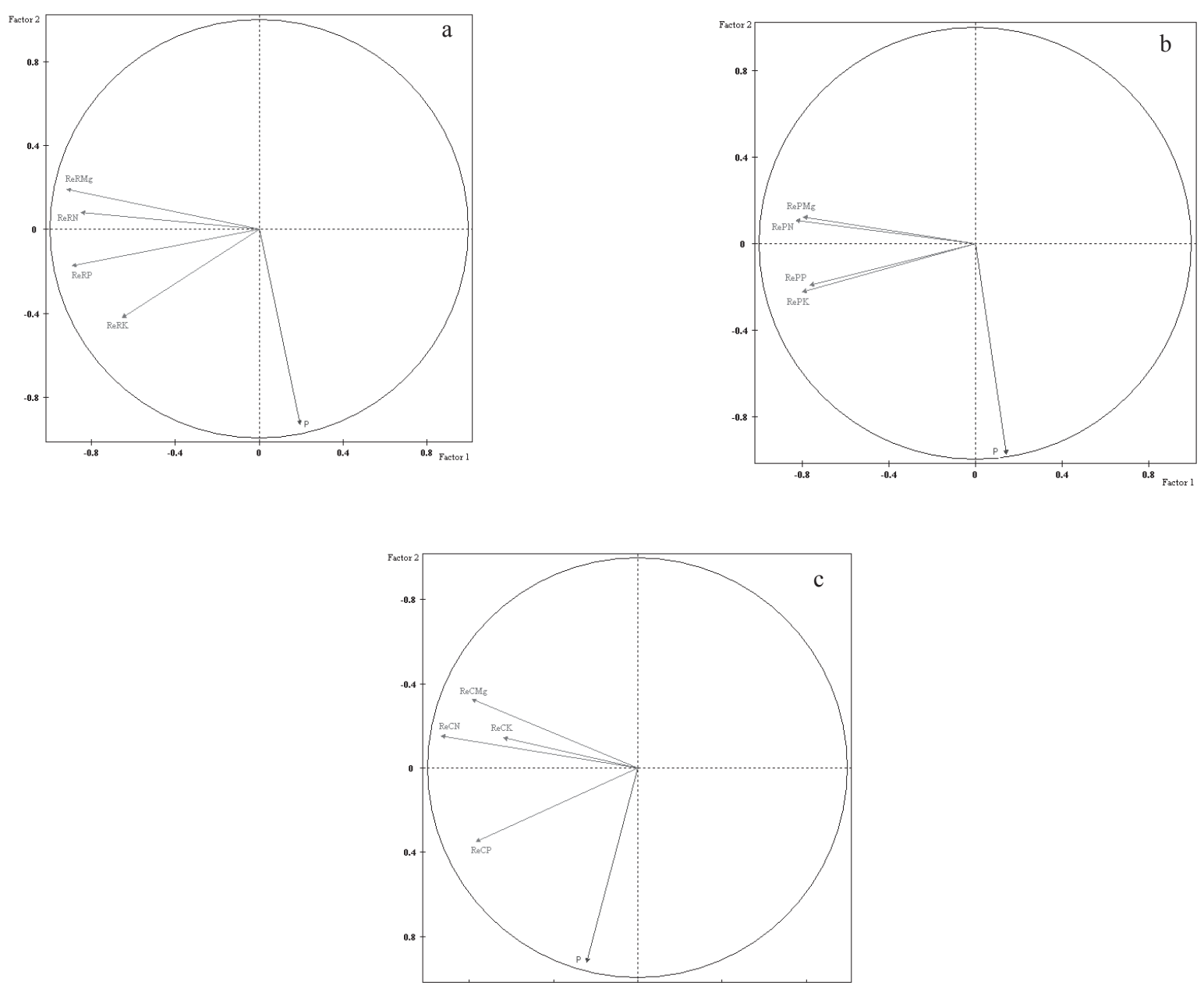

Figura 2. Primer plano factorial del ACP para las tres especies: a: roble, b: pino, c: ciprés.

de dispersión relativamente amplio y similar, sin tendencia definida. El mayor IEV para P (Tabla 9) se registró en roble, siendo similares entre sí los de las coníferas. Las diferencias entre las especies no fueron grandes y los valores altos, lo cual presupone una economía estrecha en el uso del nutriente dada su baja disponibilidad en el suelo. Las gráfi- cas de IEv frente a la cantidad de $\mathrm{P}$ en la necromasa (Figuras 3 a 5) mostraron patrones relativamente similares de eficiencia para las tres especies. La mayor eficiencia en el uso de $\mathrm{K}$ y Mg se encontró en las coníferas, alcanzándose los máximos absolutos para estos nutrientes en pino y ciprés respectivamente.

Tabla 6. Vectores propios del ACP para retranslocación por especie.

\begin{tabular}{lccccc}
\hline Especie & Variable & Eje 1 & Eje 2 & Eje 3 & Eje 4 \\
\hline \multirow{4}{*}{ Roble } & ReRN & -0.85 & 0.08 & -0.21 & 0.47 \\
& ReRMg & -0.92 & 0.19 & -0.15 & -0.16 \\
& ReRK & -0.65 & -0.42 & 0.63 & 0.06 \\
& ReRP & -0.89 & -0.17 & -0.17 & -0.31 \\
& $P$ & 0.19 & -0.93 & -0.30 & 0.04 \\
\hline
\end{tabular}




\begin{tabular}{ccccccc}
\hline Especie & Variable & Eje 1 & Eje 3 & Eje 3 & Eje 4 & \\
\hline \multirow{6}{*}{ Pino } & RePN & -0.83 & 0.11 & -0.33 & 0.08 & \\
& RePMg & -0.79 & 0.12 & 0.31 & -0.51 & \\
& RePK & -0.80 & -0.22 & -0.40 & 0.01 & \\
& RePP & -0.76 & -0.19 & 0.46 & 0.41 & \\
\hline \multirow{6}{*}{ Pino } & $P$ & 0.14 & -0.97 & 0.00 & -0.14 & \\
& RePN & -0.83 & 0.11 & -0.33 & 0.08 & -0.43 \\
& RePMg & -0.79 & 0.12 & 0.31 & -0.51 & 0.00 \\
& RePK & -0.80 & -0.22 & -0.40 & 0.01 & 0.39 \\
& RePP & -0.76 & -0.19 & 0.46 & 0.41 & 0.03 \\
Ciprés & $P$ & 0.14 & -0.97 & 0.00 & -0.14 & -0.14 \\
& ReCN & 0.93 & -0.15 & -0.04 & -0.02 & 0.32 \\
& ReCMg & 0.78 & -0.33 & 0.36 & -0.35 & -0.17 \\
& ReCK & 0.64 & -0.15 & -0.74 & 0.06 & -0.15 \\
& ReCP & 0.77 & 0.35 & 0.33 & 0.41 & -0.10 \\
& $P$ & 0.24 & 0.92 & -0.12 & -0.27 & 0.01 \\
\hline
\end{tabular}

ReRN: retranslocación roble nitrógeno, ReRMg: retranslocación roble magnesio, ReRK: retranslocación roble potasio, ReRP: retranslocación roble fósforo, RePN: retranslocación pino pátula nitrógeno, RePMg: retranslocación pino pátula magnesio, RePK: retranslocación pino pátula potasio, RePP: retranslocación pino pátula fósforo, ReCN: retranslocación ciprés nitrógeno, $\mathrm{ReCMg}$ : retranslocación ciprés magnesio, ReCK: retranslocación ciprés potasio, ReCP: retranslocación ciprés fósforo, P: precipitación.

\section{DISCUSIÓN DE RESULTADOS}

\section{CONCENTRACIONES FOLIARES}

La proporcionalidad encontrada hasta cierto punto para las tres especies entre las concentraciones de $\mathrm{N}$ en la hojarasca foliar y en las hojas maduras sugirió un comportamiento similar en cuanto a su retranslocación. Los valores de $\mathrm{N}$ en las hojas maduras, así como en la necromasa foliar, se correspondieron con las reportadas por otros estudios en bosques montanos tropicales (13.4-17.8 $\mathrm{mg} \mathrm{N} \mathrm{g}^{-1}$ : Steinhardt 1979, Fassbender \& Grimm 1981, Proctor et al. 1989, Veneklaas 1991). Las concentraciones de $\mathrm{N}$ en la hojarasca foliar de pino fueron superiores a las reportadas por Lundgren (1978) en Tanzania para esta especie, y más del doble de las verificadas en plantaciones de $P$. caribaea y $P$. elliottii de Puerto Rico (Cuevas \& Lugo 1998). Las bajas concentraciones de $\mathrm{N}$ en las hojas maduras de ciprés no se correspondieron con las reservas del suelo (Tabla 1), las cuales doblaron las encontradas en el robledal y sugirieron el carácter no limitante del nutriente para la primera.
Las concentraciones de $\mathrm{P}$, tanto en las hojas maduras como en las que componen la hojarasca foliar, se situaron en los límites inferiores reportados para otros bosques montanos tropicales. Para hojas maduras Veneklaas (1991) encontró concentraciones de $\mathrm{P}$ de $0.9 \mathrm{mg} \mathrm{P} \mathrm{g}^{-1}$, mientras que Vera et al. (1999), para un grupo de diez especies altoandinas, reportaron valores en el rango 0.8-1.9 mg $\mathrm{P}$ $\mathrm{g}^{-1}$, cantidades que no se alcanzaron en ninguno de nuestros bosques. Las concentraciones de $\mathrm{P}$ de la hojarasca foliar aquí verificadas caen en el límite inferior del intervalo reportado por numerosos estudios en bosques montanos tropicales $(0.2-1.0 \mathrm{mg}$ $\left.\mathrm{P} \mathrm{g}^{-1}\right)$. Para los andisoles del Oriente Antioqueño ha sido señalada una baja disponibilidad de P (Tschinkel 1972, Sánchez 1976, Rodríguez \& Molina 1990, Jaramillo 1995), ocasionada por la inmovilización de fosfatos solubles por minerales amorfos alofánicos. Esta alta capacidad de fijación de P influye en las bajas concentraciones foliares, así como en el desarrollo de estrategias de conservación del nutriente (Londoño et al. 2005, Ramírez et al. 2007). Al parecer $P$ ejerce un papel limitante 
para las especies aquí estudiadas, conforme lo sugieren sus bajos valores en el suelo (Tabla 1).

Las concentraciones de $\mathrm{Ca}$ en la hojarasca foliar de pino y ciprés fueron superiores a las reportadas para otras plantaciones del género Pinus (2.9 - 3.3 mg Ca $\mathrm{g}^{-1}$ : Cuevas \& Lugo 1998). Los máximos absolutos de concentración de $\mathrm{Ca}$ en la hojarasca foliar se encontraron en ciprés, en cuyos rodales se presentaron además las mayores reservas edáficas de $\mathrm{Ca}$ (Tabla 1). Las concentraciones de $\mathrm{Mg}$ en la hojarasca foliar se ubicaron en el límite inferior del intervalo definido para un amplio número de bos- ques tropicales de tierras altas (1.2-3.0 $\left.\mathrm{mg} \mathrm{Mg} \mathrm{g}^{-1}\right)$, propiciadas por el origen volcánico del suelo y por los bajos valores de $\mathrm{pH}$, donde priman los iones $\mathrm{H}^{+}$. Los bajos valores de $\mathrm{Mg}$ en la hojarasca foliar de ciprés, en donde se encontraron además los máximos de $\mathrm{Ca}$, sugirieron la ocurrencia de un desequilibrio $\mathrm{Ca} / \mathrm{Mg}$, según el cual se ve favorecida la absorción del primero ante su mayor abundancia en el suelo y la ocurrencia de valores mayores de $\mathrm{pH}$. Estos bajos valores podrían asimismo reflejar una retranslocación más intensa del nutriente en ciprés.
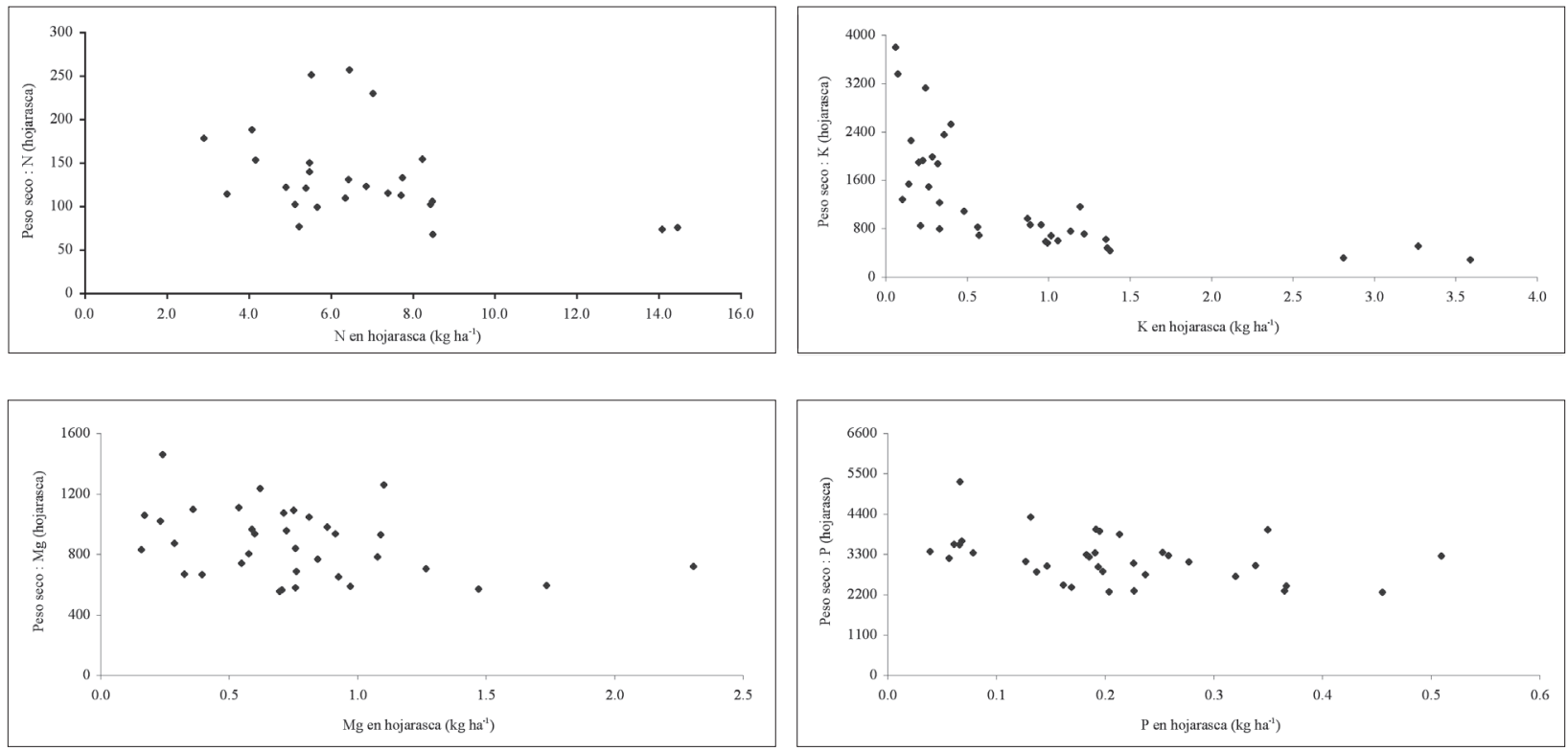

Figura 3. Relación entre la razón peso seco: nutriente y cantidad de nutriente en la hojarasca fina de roble.
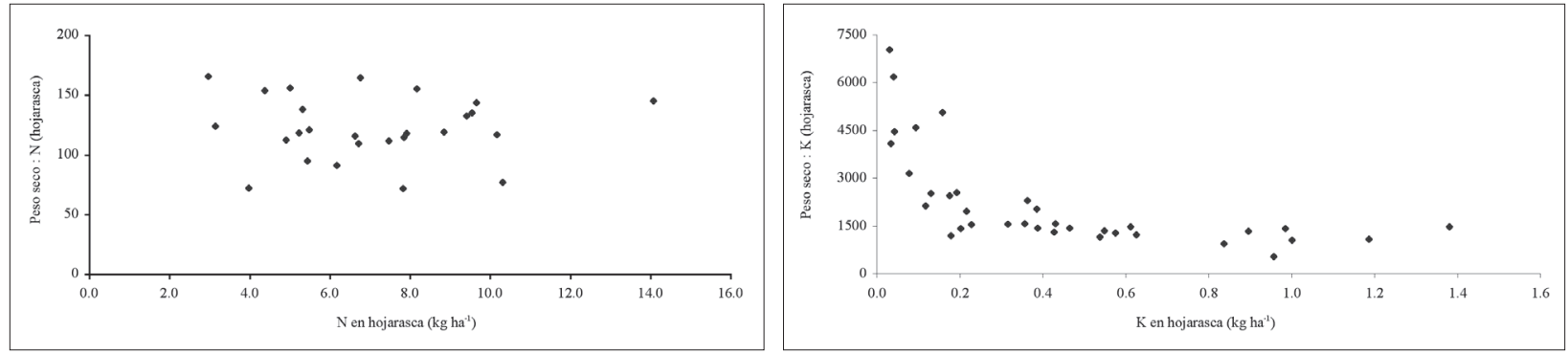

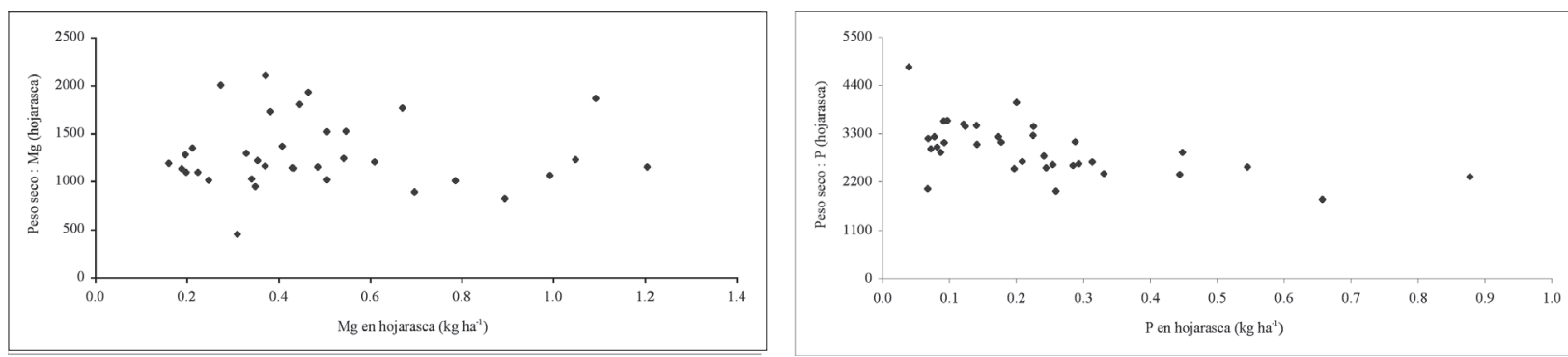

Figura 4. Relación entre la razón peso seco: nutriente y la cantidad de nutriente en la hojarasca fina de pino.
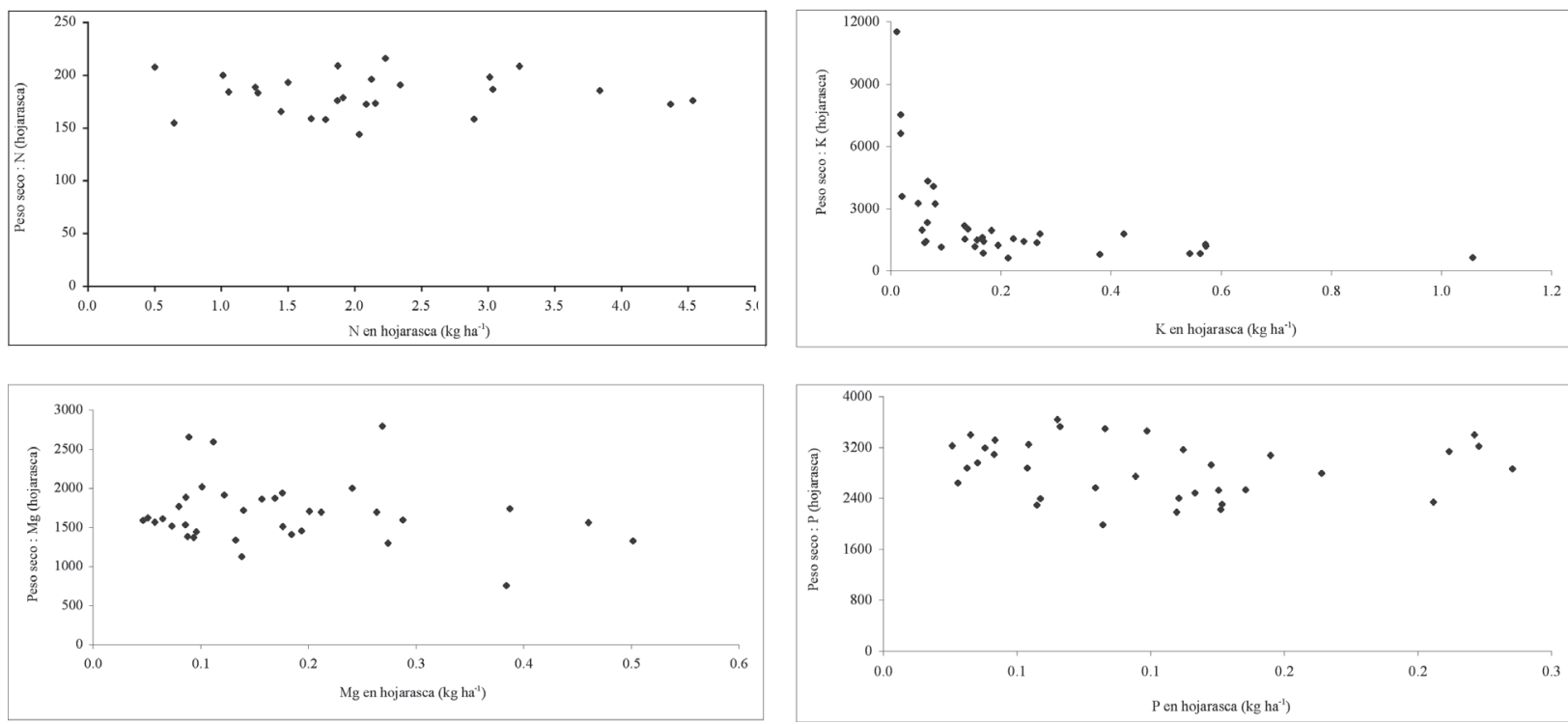

Figura 5. Relación entre la razón peso seco: nutriente y la cantidad de nutriente en la hojarasca fina de ciprés.

Las concentraciones de $\mathrm{K}$ en las hojas maduras fueron muy similares entre especies $(2.7-3.6 \mathrm{mg}$ de $\mathrm{K} \mathrm{g}^{-1}$ ), ubicándose incluso por debajo de los límites inferiores reportados por otros estudios en bosques montanos tropicales (30 - $50 \mathrm{mg}$ de $\mathrm{K} \mathrm{g}^{-1}$ : Medina et al. 1981, Proctor et al. 1989). Las concentraciones de K en la hojarasca foliar de roble se correspondieron con las de otros bosques tropicales de tierras altas (Proctor et al. 1989, Vitousek et al. 1995). Por otra parte, en los rodales de pino y ciprés estudiados se encontraron valores foliares de $\mathrm{K}$ similares a los obtenidos por Lundgren (1978) para plantaciones de $C$. lusitanica $\left(0.8 \mathrm{mg}\right.$ de $\left.\mathrm{K} \mathrm{g}^{-1}\right)$ e inferiores para $P$. patula $\left(1.4 \mathrm{mg}\right.$ de $\left.\mathrm{K} \mathrm{g}^{-1}\right)$. Nuestros valores de $\mathrm{K}$ en la hojarasca foliar se ubican en el extre- mo inferior del intervalo pantropical sugerido por Duivenvoorden \& Lips (1995) de $0.27 \pm 0.11(\% \pm$ desviación típica). Si bien es necesario considerar otras rutas de circulación, la eventual escasez del elemento alcalino podría afectar la productividad primaria de la comunidad, ya que éste no puede ser reemplazado por otro en sus funciones esenciales del metabolismo de las plantas: activación de numerosas enzimas, control estomático, fotosíntesis y síntesis de proteínas, entre otras (Malavolta 1984, Del Valle 2003). Los resultados podrían asimismo estar reflejando el conocido antagonismo entre $\mathrm{Ca}$ y $\mathrm{K}$, según el cual la absorción de iones $\mathrm{K}^{+}$se ve suprimida por la abundancia de cationes $\mathrm{Ca}^{2+}$ (Baule \& Fricker 1970, Binkley 1993), lo cual, propor- 
cionalmente, podría deducirse de lo encontrado en ciprés para ambos nutrientes.

\section{RETRANSLOCACIÓN DE NUTRIENTES}

La retranslocación se relacionó de forma inversa con la cantidad de lluvia, como consecuencia del lavado foliar. La comparación de los valores de retranslocación obtenidos a nivel de especie con otros estudios realizados en bosques tropicales es compleja, ya que la mayoría de éstos han obtenido los índices de retranslocación a partir de comparaciones entre hojas maduras y hojarasca a nivel del bosque (Vera et al. 1999).

Así, el intervalo de valores de retranslocación de $\mathrm{N}$ originado a partir de los reportes de estudios realizados en bosques montanos tropicales (Tanner 1977, Grubb \& Edwards 1982, Cuevas \& Lugo 1998, Vera et al. 1999) oscila entre $14 \%$ y $70 \%$, intervalo de mayor amplitud que el que se obtiene a partir de datos provenientes de estudios realizados en bosques tropicales de tierras bajas (Medina 1984, Vitousek \& Sanford 1986, Scott et al. 1992, Villela \& Proctor 1999, Chuyong et al. 2000), en donde se esperarían menores limitaciones por $\mathrm{N}$. Los valores de retranslocación de $\mathrm{N}$ para roble $(35.6 \%)$ y pino $(37.0 \%)$ se acercaron al promedio calculado (43.3\%) a partir de los datos de Vera et al. (1999) para diez especies arbóreas de un bosque montano alto de Colombia. Los mínimos absolutos anuales de $\mathrm{N}$ retranslocados se registraron en el cipresal, siendo incluso inferiores a los reportados para bosques templados de Castanea sativa $(9.4 \mathrm{~kg}$ $\mathrm{N} \mathrm{ha}^{-1}$ año $^{-1}$ : Santa Regina et al. 2001).

La menor retranslocación de $\mathrm{N}$ en ciprés, y sus altas reservas edáficas, sugirieron su carácter no limitante para la especie; esto podría explicar parcialmente la falta de correspondencia entre las concentraciones foliares y su reserva edáfica. Al respecto se ha expuesto que una especie con baja concentración foliar de un nutriente determinado podría ser usada como un indicador estándar de baja disponibilidad de aquel en un sitio concreto (Vitousek 1982). Ya que no es éste el caso, bien podría indicar una baja demanda por la especie o, aún ante cantidades totales altas del nutriente, la imposibilidad de ser tomado por la planta.
La máxima retranslocación de $\mathrm{P}$ se encontró para roble, seguida de lejos por pino y ciprés. Si bien ha sido aceptada la teoría de que la retranslocación de $\mathrm{P}$ podría tener un significado adaptativo para las especies vegetales en suelos derivados de cenizas volcánicas dada su adsorción por las alofanas (Vitousek \& Sanford 1986), también se ha reportado que su retranslocación no varía en forma consistente con su disponibilidad (Vera et al. 1999). La relación inversa encontrada entre la retranslocación de $\mathrm{P}$ y la lluvia pudo deberse al proceso de absorción foliar en el dosel, conforme lo encontró León (2007) en estos mismos sitios. Así, los valores de deposición neta o pluviolavado (calculada para cada nutriente como deposición neta $(\mathrm{Dn})=$ Precipitación interna + Escorrentía cortical - Precipitación total), que representan la magnitud de las transferencias de nutrientes por lavado foliar, fueron negativos en los tres bosques $\left(0.6 \mathrm{~kg} \mathrm{P} \mathrm{ha}^{-1}\right.$ año ${ }^{-1}$ en promedio), que favorecerían incrementos en las concentraciones foliares, afectando por ende los cálculos de retranslocación.

Los valores medios de la relación $\mathrm{P}: \mathrm{N}$ de las hojas maduras (Tabla 3) de roble y pino (0.03 ambas) indicaron una baja disponibilidad de $\mathrm{P}$ edáfico (Medina et al. 1990) y, en el caso de ciprés (0.05), un umbral de aceptación escasamente superado. La retranslocación media de $\mathrm{P}$ en roble $(31.4 \%)$ se corresponde con las reportadas por otros estudios en bosques montanos tropicales (Vera et al. 1999), así como con los indicados por Cavelier (1996) para bosques tropicales de tierras bajas (29.0 - 50.0\%), en donde usualmente se presentan limitaciones de $P$ para la nutrición vegetal. Por el contrario, la restranslocación de $\mathrm{P}$ en las coníferas fue muy baja (12.4 - 18.5\%), reflejando su menor dependencia de este proceso para satisfacer las demandas del nutriente. Las estructuras ecto y endomicorrizales son de crucial importancia en el ciclo de P, y podrían explicar la situación descrita, ya que han sido verificadas en las plantaciones de pino y ciprés de la zona (Alvarado 1988a, b; León et al. 2008). Los resultados indicados coinciden con los de Huang et al. (2007), según los cuales se presenta una mayor retranslocación de $\mathrm{P}$ en las especies deciduas que en las siempreverdes. 
Aquellos bosques con una alta eficiencia en la retranslocación de $\mathrm{N}$ también presentan una alta eficiencia en la retranslocación de $\mathrm{P}$ (Martín et al. 1996), lo cual se verificó con el análisis de regresión (Tabla 7). El mejor modelo se encontró para roble, para el que ocurrieron los máximos absolutos de retranslocación de $\mathrm{P}$, como especie más exigente del nutriente. Los menores ajustes fueron para pino, que mostró menor proporcionalidad entre la retranslocación de $\mathrm{N}$ y $\mathrm{P}$. Aunque se ha indicado que en promedio las plantas perennes retranslocan más de la mitad de las existencias foliares de $\mathrm{N}$ y $\mathrm{P}$ durante la senescencia foliar (Van Heerwaarden et al. 2003), esta situación no se encontró aquí.

Los mínimos absolutos de retranslocación de $\mathrm{K}$ encontrados para roble no se correspondieron con la disponibilidad edáfica del nutriente, ya que fue precisamente en este bosque donde se encontraron las menores reservas edáficas de K (Tabla 1). Dada la alta similaridad entre especies en las concentraciones foliares de $\mathrm{K}$, y el mayor efecto del pluviolavado encontrado para roble, que producirían menores índices de retranslocación, es muy posible que el $\mathrm{K}$ sea más demandado por esta especie. La baja retranslocación de $\mathrm{K}$ podría ser resultado de la mayor fuerza que tiene en este bosque el pluvio- lavado del nutriente. La Dn reportada para los tres bosques en estudio (León 2007) fue muy superior para el robledal $\left(35.2 \mathrm{~kg} \mathrm{~K} \mathrm{ha}^{-1} \mathrm{año}^{-1}\right)$ que para las coníferas (19 $\mathrm{kg} \mathrm{K} \mathrm{ha}^{-1}$ año $^{-1}$ en promedio).

Las pérdidas de $\mathrm{K}$ podrían ser compensadas por una eficaz recaptura en el suelo, dada la alta reserva edáfica del robledal, que fue cercana a la mitad de la de las coníferas, aunque en ellas el pluviolavado fue, por contrapartida, muy inferior. Así, es probable que en el robledal ocurra un reciclaje directo y cerrado (Chuyong et al. 2000), estando las raíces finas y las hifas de hongos muy cerca de la hojarasca. El mantillo podría constituir una trampa eficiente para nutrientes mineralizados de la hojarasca y aquellos (especialmente $\mathrm{K}$ y $\mathrm{Mg}$ ) lavados del dosel. La masa de raíces finas en el mantillo de roble fue cercana a $4 \mathrm{Mg} \mathrm{ha}^{-1}$, valor 4 veces superior al del pinar y 2.5 veces al del cipresal (León 2007). La retranslocación de K para roble (39.1\%) se correspondió de cerca con la reportada por Veneklaas (1991) para bosques montanos de Colombia (34\%). Sin embargo, no es conveniente definir patrones de retranslocación para este nutriente con base en otros estudios, dadas las diferencias en las frecuencias del muestreo y el marcado carácter lixiviable de K (Tukey 1970).

Tabla 7. Modelos de regresión lineal ajustados para el P en función del $\mathrm{N}$ retranslocado por especie.*

\begin{tabular}{cccc}
\hline Especie & Modelo & $\mathbf{R}^{2}(\%)$ & $\mathbf{r}$ \\
\hline Roble & ReRP $=-24.1938+1.46631^{*}$ ReRN & 63.40 & 0.80 \\
Pino & $\operatorname{RePP}=27.2825+0.425797^{*}$ RePN & 26.02 & 0.51 \\
Ciprés & ReCP $=5.82702+0.761567^{*}$ ReCN & 49.20 & 0.70 \\
\hline *Todos los modelos significativos $(\mathrm{P}<0.05)$ &
\end{tabular}

La retranslocación de $\mathrm{Mg}$ para ciprés fue de más del doble de magnitud que la de las otras dos especies, cuyos valores fueron similares. En el robledal muy probablemente este bajo valor fue resultado del lavado foliar del nutriente, el cual siguió la secuencia decreciente (León 2007): robledal $(1.8 \mathrm{~kg}$ $\left.\mathrm{Mg} \mathrm{ha}^{-1} \mathrm{año}^{-1}\right)>\operatorname{pinar}\left(1.1 \mathrm{~kg} \mathrm{Mg} \mathrm{ha}{ }^{-1} \mathrm{año}^{-1}\right)>$ cipresal $\left(0.8 \mathrm{~kg} \mathrm{Mg} \mathrm{ha}^{-1} \mathrm{año}^{-1}\right)$. Las menores concentraciones de $\mathrm{Mg}$, indicativas a su vez de menores demandas, se determinaron para ciprés, así como las menores pérdidas por lavado y los mayores contenidos edáficos; la mayor retranslocación encontrada sugiere pues la ocurrencia de un reciclaje más intenso a través de esta vía que a través de la del sistema suelo-planta, conforme ha sido indicado (Santa Regina et al. 2001) para nutrientes en bajas cantidades en suelos volcánicos. La alta retranslocación de $\mathrm{Mg}$ podría reflejar una estrategia de la especie para regular la relación $\mathrm{Mg} / \mathrm{Ca}$ (Proctor et al. 1989), que en hojas maduras fue de 0.1 para ciprés, mientras que para roble y pino fue de 0.4. La cantidad de Mg retranslocada (Tabla 4) 
Tabla 8. Matriz de correlación lineal para retranslocación de elementos de las tres especies.

\begin{tabular}{|c|c|c|c|c|c|c|}
\hline Especie & Variable & $\operatorname{ReN}$ & ReMg & ReK & ReP & $\mathbf{P}$ \\
\hline \multirow{5}{*}{ Roble } & ReN & 1.00 & & & & \\
\hline & ReMg & $0.73^{*}$ & 1.00 & & & \\
\hline & ReK & 0.41 & 0.42 & 1.00 & & \\
\hline & $\operatorname{ReP}$ & $0.65^{*}$ & $0.80^{*}$ & $0.52^{*}$ & 1.00 & \\
\hline & $\mathrm{P}$ & -0.16 & -0.30 & 0.08 & 0.01 & 1.00 \\
\hline \multirow{5}{*}{ Pino } & ReN & 1.00 & & & & \\
\hline & ReMg & $0.53^{*}$ & 1.00 & & & \\
\hline & ReK & $0.60^{*}$ & $0.48^{*}$ & 1.00 & & \\
\hline & ReP & $0.48^{*}$ & $0.51^{*}$ & $0.49^{*}$ & 1.00 & \\
\hline & $P$ & -0.17 & -0.16 & 0.04 & 0.02 & 1.00 \\
\hline \multirow{5}{*}{ Ciprés } & ReN & 1.00 & & & & \\
\hline & ReMg & $0.72^{*}$ & 1.00 & & & \\
\hline & ReK & $0.60^{*}$ & 0.28 & 1.00 & & \\
\hline & ReP & $0.61^{*}$ & $0.48^{*}$ & 0.23 & 1.00 & \\
\hline & $P$ & 0.10 & -0.06 & 0.09 & 0.36 & 1.00 \\
\hline
\end{tabular}

* Valores estadísticamente significativos $(\mathrm{P}<0.05)$.

señaló la fuerza del proceso en el cipresal, ya que a pesar de la baja caída de hojarasca (Tabla 9) que afecta dicho cálculo, la retranslocación de $\mathrm{Mg}$ fue casi de la misma magnitud que la de pino (0.77 vs. $0.80 \mathrm{~kg} \mathrm{Mg} \mathrm{ha}^{-1}$ año $^{-1}$, respectivamente), en donde por contrapartida ocurrió la mayor caída de hojarasca.

\section{EFICIENCIA EN EL USO DE NUTRIENTES}

Aunque ciprés mostró la mayor eficiencia en el uso de $\mathrm{N}$, a nivel del rodal se presentó la menor retranslocación de N. Las bajas concentraciones de $\mathrm{N}$ en las hojas maduras y los mayores contenidos edáficos sugirieron bajas demandas de $\mathrm{N}$ por ciprés o dificultades para su captura. No obstante, no fue evidente la baja disponibilidad de N, ya que, de acuerdo con los resultados de León et al. (2008) en los mismos sitios de estudio, en el cipresal el número de colonias de bacterias fijadoras de $\mathrm{N}$ presentes en el suelo ( $100 \times 10^{5} / \mathrm{g}$ de suelo) fue muy superior al de las otras coberturas. Además la tasa de mineralización de $\mathrm{N}$ (3.9 $\mathrm{mg} \mathrm{NH}_{4}^{+} \mathrm{kg}^{-1}$ suelo día ${ }^{-1}$ ) fue casi de igual magnitud a la máxima registrada en el robledal (4.1 mg NH${ }_{4}^{+} \mathrm{kg}^{-1}$ suelo día ${ }^{-1}$ ). Dado que el cálculo del IEV está afectado por el lavado y/o la absorción que puede ocurrir en el dosel, es difícil precisar con exactitud el fenómeno. Los valores IEV de $\mathrm{N}$ reportados por Lundgren (1978) para plantaciones de $C$. lusitanica y $P$. patula de Tanzania (119 y 152) son, en el caso de la primera especie, inferiores a los nuestros (187), mientras que para la segunda son un poco mayores (123).

Tabla 9. Índices de eficiencia en el uso de nutrientes para las tres especies. Valores expresados en $\mathrm{kg} \mathrm{ha}^{-1} \mathrm{año}^{-1}$.

\begin{tabular}{cccccccc}
\hline Especie & Parámetro & Materia seca & N & P & Ca & Mg & K \\
\hline \multirow{2}{*}{ Roble } & Desfronde & 7498 & 78.4 & 2.4 & 44.5 & 9.0 & 9.8 \\
& IEV & & 95.7 & 3101 & 168.7 & 835.4 & 764.5 \\
\multirow{2}{*}{ Pino } & Desfronde & \multirow{2}{*}{7768} & 63.1 & 2.8 & 27.2 & 6.0 & 5.2 \\
& IEV & & 123 & 2804 & 285.5 & 1304.9 & 1502 \\
\multirow{2}{*}{ Ciprés } & Desfronde & \multirow{2}{*}{3488} & 18.7 & 1.2 & 34.8 & 1.9 & 2.5 \\
& IEV & & 186 & 2947 & 100 & 1850.1 & 1377 \\
\hline
\end{tabular}

IEV: índice de eficiencia de Vitousek (1982). 
En el pinar se verificó una mayor eficiencia en el uso de $\mathrm{N}$ que en el robledal, aunque en ambos bosques fueron similares los contenidos de $\mathrm{N}$ edáfico y foliar, así como una retranslocación del nutriente en proporciones similares, indicando menor demanda de $\mathrm{N}$ por la conífera. El valor IEV obtenido para roble se corresponde con el calculado, a su vez, a partir de los datos de Veneklaas (1991) para un bosque montano bajo de Risaralda (85.5). Los valores IEV para pino y roble fueron similares a los reportados para bosques tropicales de tierras bajas de Peltogyne gracilipes (105 para la hojarasca foliar y 93 para la hojarasca total, Villela \& Proctor 1999), así como a los reportados para bosques también del Amazonas brasilero (Smith et al. 1998) de 84.8 (bosque natural) y de 96.5 (plantación de Carapa guianensis). Estos últimos autores expusieron que el bosque natural y la plantación de leguminosas, que presentaron un uso ineficiente de N, mostraron por contrapartida altas tasas de mineralización de $\mathrm{N}$ en la superficie del suelo -aspecto ampliamente reconocido en las especies leguminosas- en comparación con las especies que exhibieron una mayor eficiencia en el uso de N. La especie de conífera estudiada por ellos ( $P$. caribaea) fue la que mayor eficiencia mostró en el uso de N (IEV $=238)$. Un valor similar (alrededor de 220) fue encontrado para esta especie en Puerto Rico (Cuevas \& Lugo 1998).

Los gráficos de EUN vs. contenido de nutriente en la hojarasca (Figuras 3 a 5), muestran que para contenidos de $\mathrm{N}$ en la necromasa entre 3.0 y $9.0 \mathrm{~kg} \mathrm{~N}$ $\mathrm{ha}^{-1}$, la Eun oscila entre 60 y 250 para roble y 70 y 170 para pino, valores que caen en el intervalo reportado para EUN de bosques montanos tropicales $(80$ - 180, Vitousek 1984). Este valor IEV cercano a 60 coincide con el sugerido por Vitousek (1982) como valor mínimo para un número amplio de bosques tropicales a partir de hojarasca fina, el cual podría representar la cantidad máxima de $\mathrm{N}$ no hidrolizable, y por tanto inmóvil, que los árboles pueden acumular. Cabe destacar que este valor mínimo es cercano a 150 en ciprés, lo que apoyaría parcialmente la menor retranslocación de $\mathrm{N}$ allí encontrada. En el cipresal se registró asimismo la menor cantidad de $\mathrm{N}$ retornada vía hojarasca foliar y los mayores IEV, de manera similar a como lo re- portó Vitousek (1984). Este autor planteó, además, que en promedio la eficiencia de la producción de hojarasca por unidad de $\mathrm{N}$ es mayor en bosques de coníferas que en los bosques tropicales.

Las ligeras diferencias aquí encontradas en la eficiencia en el uso de P entre especies (Tabla 9) y los elevados valores de los IEV sugieren una economía estrecha en el uso de $\mathrm{P}$, dada su baja disponibilidad edáfica. Así, los similares contenidos totales de $\mathrm{P}$ en el suelo de los tres bosques (en promedio $4 \mathrm{~kg} \mathrm{Pha}^{1}$ ) permiten señalar una muy alta eficiencia en comparación con la reportada por otros autores (Grimm \& Fassbender 1981, Edwards \& Grubb 1982) para bosques montanos tropicales. De hecho, los valores negativos de deposición neta (Dn) o pluviolavado encontrados por León (2007) señalan la absorción de $\mathrm{P}$ en el dosel (cipresal: $-0.8 \mathrm{~kg} \mathrm{P} \mathrm{ha}^{-1} \mathrm{año}^{-1}$, pinar: $-0.6 \mathrm{~kg} \mathrm{P} \mathrm{ha}^{-1} \mathrm{año}^{-1}$, robledal: $-0.5 \mathrm{~kg} \mathrm{P} \mathrm{ha}^{-1} \mathrm{año}^{-1}$ ). Según Vitousek (1984), algunos bosques montanos tropicales muestran una alta eficiencia a nivel del rodal en el uso de $\mathrm{P}$, que se refleja en su circulación en la hojarasca en cantidades inferiores a $3 \mathrm{~kg} P$ $\mathrm{ha}^{-1}$ año ${ }^{-1}$, cifra que fue inferior en los tres bosques (cipresal: $1.2 \mathrm{~kg} \mathrm{P} \mathrm{ha}{ }^{-1} a_{n} o^{-1}$, robledal: $2.4 \mathrm{~kg} \mathrm{Pha}^{1}$ año ${ }^{-1}$, pinar: $2.8 \mathrm{~kg} \mathrm{P} \mathrm{ha}^{-1} \mathrm{año}^{-1}$ ) y que supone un uso eficiente del nutriente.

Tanto para bosques tropicales de tierras bajas como de tierras altas ha sido señalado (Silver 1994) que a menores contenidos edáficos de $\mathrm{P}$ (total y extraíble) se obtienen mayores valores de IEv, lo que indica su carácter limitante para el crecimiento en ambos biomas y en nuestro caso particular para los tres bosques estudiados. Nuestros valores IEV para P oscilaron entre 3101 en el robledal y 2804 en el pinar, ocupando un lugar intermedio entre los reportados para otros bosques montanos tropicales. Los menores valores (1000) se han encontrado en bosques de Tanzania, localizados a $1400 \mathrm{msnm}$ (Lundgren 1978); de Colombia (1153), a $2550 \mathrm{msnm}$ (Veneklaas 1991), y de Venezuela (1613), a 2300 msnm (Steinhardt 1979). Los mayores valores IEV corresponden, por otra parte, a los bosques de Sarawak (3846), a una elevación de $1860 \mathrm{msnm}$ (Proctor et al. 1983) y a los montanos de formaciones insulares de Sabah, entre 5000 y 5882 (Proctor et al. 1989) y de Puerto Rico (4167, Medina et al. 1981). 
El mayor IEV de P de roble se correspondió con la mayor retranslocación de $\mathrm{P}$, lo cual sugiere que la especie (dadas las similares condiciones de concentración foliar entre especies y contenidos edáficos entre coberturas) acude a esta estrategia quizás no sólo por la posible fijación de $\mathrm{P}$ en el suelo, sino además por la competencia entre especies en el bosque. De las gráficas de IEV frente a la cantidad de $\mathrm{P}$ en la necromasa (Figuras 2 a 5) se observaron patrones relativamente similares de eficiencia para los tres bosques. En el gráfico de dispersión los puntos se ubicaron por encima y por debajo de 3000 de manera relativamente consistente, con lo cual se puede señalar (Weerakkody \& Parkinson 2006) que las tres especies realizan un uso eficiente de P. En el caso de ciprés se estableció una ligera diferencia determinada por la cantidad de $\mathrm{P}$ que circuló en la hojarasca, mucho más estrecha que la de las otras dos coberturas; sin embargo, para las tres, el valor mínimo del índice fue cercano a 2000, un valor casi dos veces mayor al mínimo encontrado por Vitousek (1982).

La especie más eficiente en el uso de K según el IEV fue pino, seguida de cerca por ciprés y luego por roble, esta última con valores claramente inferiores a los de las especies de coníferas. Sin embargo, es necesario resaltar la importancia que tiene el lavado en la circulación de este nutriente, y que fue en el robledal donde se registró el mayor pluviolavado (43.5 $\left.\mathrm{kg} \mathrm{K} \mathrm{ha}^{-1} \mathrm{año}^{-1}\right)$ con relación a las plantaciones de coníferas ( 26.1 y $\left.27.6 \mathrm{~kg} \mathrm{~K} \mathrm{ha}^{1} \mathrm{año}^{-1}\right)$. Los valores del IEV para K en roble (764) se acercaron a los de bosques montanos tropicales de Puerto Rico (714, Medina et al. 1981) y de Sabah (1000-1111, Proctor et al. 1989). Fueron, por otra parte, muy superiores a los obtenidos en bosques montanos tropicales de Colombia (118-309, Veneklaas 1991). Valores también muy bajos (167-181) se reportaron para bosques tropicales de tierras bajas del Amazonas brasilero por Villela \& Proctor (1999). Las deficiencias que parecieran existir en el pinar, a juzgar por su escaso contenido y forma de la curva (Figura 3), podrían explicarse por la mayor producción de materia seca por desfronde y crecimiento y por el mínimo absoluto para Dn de K entre los tres bosques (26.1 $\mathrm{kg} \mathrm{K} \mathrm{ha}^{-1}$ año $^{-1}$, León 2007).
La baja eficiencia en el uso de Ca por ciprés (IEV $=$ 100) se correspondió con su elevada concentración foliar, que supone, dado su carácter poco móvil, una elevada absorción y su puesta en circulación vía hojarasca. Dicho valor fue muy inferior al encontrado por Lundgren (1978) en las plantaciones de $C$. lusitanica en condiciones edáficas más favorables. Por el contrario, la eficiencia para las plantaciones de $P$. patula de este autor (129) fue muy inferior a la aquí obtenida (285) para la misma especie. La mayor eficiencia en el uso de $\mathrm{Ca}$ en nuestra plantación de pino se correspondió además con el máximo absoluto de Dn encontrado en esta plantación (1.5 kg Ca ha ${ }^{-1}$ año ${ }^{-1}$ : León 2007), con lo cual se estaría presentando un proceso de enriquecimiento que afectaría los cálculos; no obstante, las diferencias para Dn no fueron estadísticamente significativas entre los tres bosques.

Al parecer en ciprés se presentó un desequilibrio nutricional $\mathrm{Ca} / \mathrm{Mg}$, como lo sugiere el hecho de encontrarse una eficiencia en el uso de $\mathrm{Mg}$ inversa a la de $\mathrm{Ca}$, situación que además coincidió con la mayor retranslocación de Mg. Estos factores parecen ser los más significativos para explicar la mayor EUN en este bosque, ya que allí se presentaron los mínimos absolutos de Dn $\left(0.8 \mathrm{~kg} \mathrm{Mg} \mathrm{ha}^{-1}\right.$ año ${ }^{1}$ : León 2007), con lo cual pierde importancia el efecto del lavado foliar. La eficiencia en el uso de $\mathrm{Mg}$ fue mayor, además, para pino que para roble. El valor IEV obtenido para esta última especie, sin embargo, fue muy superior al calculado para los bosques montanos tropicales de Tanzania (383) estudiados por Lundgren (1978), encontrándose más próximo al reportado por Villela \& Proctor (1999) para bosques tropicales de tierras bajas (391) con escasa participación de la especie Peltogyne gracilipes. El valor IEV para Mg de pino (1305) fue muy superior al obtenido para plantaciones de esta especie (444) por Lundgren (1978).

\section{CONCLUSIONES}

La EUN fue sumamente variable entre especies y nutrientes. Las coníferas presentaron una mayor eficiencia en el uso de N, Mg y K debido a su frugalidad. En el caso particular de P la eficiencia en su uso en estos suelos derivados de ceniza volcá- 
nica fue alta y relativamente similar entre las tres especies, lo que señala su carácter limitante, como de hecho lo apoyan los valores de absorción a nivel del dosel, o deposición neta negativa. Los valores de retranslocación de $\mathrm{N}$ rondaron el $50 \%$, siendo la eficiencia en su uso más baja en el robledal que en las plantaciones de coníferas. No se presentó una relación clara entre la retranslocación y la EUN, caso de K para pino, para el que ambos procesos coincidieron. El único nutriente para el que persistió una relación directa entre retranslocación y EUN fue el $\mathrm{Mg}$, verificándose una mayor EuN asociada a una mayor retranslocación, y siendo la dirección de ambos procesos ciprés $>$ pino $>$ roble. Así como puede ocurrir para los índices de eficiencia calculados a partir de la producción de hojarasca (IEV), el proceso de retranslocación resulta afectado por los procesos de absorción y lavado foliar que se producen en el dosel. Los resultados permitieron corroborar la hipótesis formulada para nutrientes fundamentales en la nutrición vegetal, como $\mathrm{N}$ y $\mathrm{P}$, de forma tal que las coníferas son menos dependientes de la retranslocación y muestran una mayor EUN que el roble. Se encontró de forma particular una retranslocación de $\mathrm{P}$ muy superior en roble, como mecanismo de conservación del nutriente desarrollado por la especie, dada su limitada disponibilidad en estos suelos derivados de cenizas volcánicas, a la cual responden las coníferas muy probablemente mediante el establecimiento de asociaciones con hongos micorrizales.

\section{AGRADECIMIENTOS}

Al Área de Biogeoquímica del Laboratorio de Ecología César Pérez Figueroa y a la Estación Forestal de Piedras Blancas de la Universidad Nacional de Colombia, Sede Medellín, por su apoyo técnico y financiero, y al personal que allí trabaja, en particular a José Vicente Betancur por su valioso concurso en el ascenso a los árboles para la toma de muestras y a Gloria Mazo por los oportunos procesamientos y determinaciones químicas en laboratorio. Agradecemos asimismo el soporte técnico y financiero prestado por el Laboratorio de Suelos del Área de Edafología y Química Agrícola de la Universidad de Salamanca, España, y a Empresas Públicas de Medellín por el acceso a los predios donde se rea- lizó el estudio y por el suministro de información meteorológica. El trabajo fue además financiado por la Dirección de Investigaciones de la Universidad Nacional de Colombia (DIME). Los autores agradecemos especialmente a los ingenieros forestales Jorge Andrés Ramírez y Claudia Marcela Zapata, quienes participaron en el establecimiento y monitoreo del ensayo.

\section{REFERENCIAS BIBLIOGRÁFICAS}

Aerts, R. \& F. S. Chapin. 2000. The mineral nutrition of wild plants revisited: a re-evaluation of processes and patterns. Advances in Ecological Research 30: 1-67.

Alvarado, B. 1988a. Determinación de micorrizas en Cupressus sp. En: F. H. Orozco (ed.). Investigaciones sobre Micorrizas en Colombia No. 2. Sociedad Colombiana de la Ciencia del Suelo. Medellín. Pp. 111-117.

Alvarado, B. 1988b. Efecto de las ectomicorrizas en el desarrollo de Pinus patula. En: F. H. Orozco (ed.). Investigaciones sobre Micorrizas en Colombia No. 2. Sociedad Colombiana de la Ciencia del Suelo. Medellín. Pp. 131-137.

Aussenac, G., M. Bonneau \& F. Le Tacon. 1972. Restitution des éléments minéraux au sol par l'intermédiaire de la litière et des précipitations dans quatre peuplements forestiers de l'Est de la France. Oecologia Plantarum 7: 1-21.

Baule, H. \& C. Fricker. 1970. The Fertilizer Treatment of Forest Trees. B .L. V. Munich.

Binkley, D. 1993. Nutrición Forestal. Prácticas de Manejo. Limusa. México D.F.

Cavelier, J. 1996. Enviromental factors and ecophysiological processes along altitudinal gradients in wet tropical mountains. En: S. S. Mulkey, R. L. Chazdon \& A. P. Smith (eds.). Tropical Forest Plant Ecophysiology. Chapman \& Hall. Nueva York. Pp. 339-439.

Chapin, F. S. 1980. The mineral nutrition of wild plants. Annual Review of Ecology and Systematics 11: 233-260. 
Charley, J. L. \& B. N. Richards. 1983. Nutrient allocation in plant communities: mineral cycling in terrestrial ecosystems. En: O. L. Lange, P. S. Nobel, C. B. Osmond \& H. Ziegler (eds.). Physiological Plant Ecology. Ecosystem Processes: Mineral Cycling, Productivity and Man's Influence. Vol IV. Springer-Verlag. Nueva York. Pp. 5-45.

Chuyong, G. B., Newbery, D. M. \& N. C. Songwe. 2000. Litter nutrients and retranslocation in a central African rain forest dominated by ectomycorrhizal trees. Research New Phytologist 148: 493-510.

Cole, D. W. \& M. Rapp. 1981. Elemental cycling in forested ecosystems. En: D. E. Reichle (ed.). Dynamic Properties of Forest Ecosystems. Cambridge University Press. Cambridge. Pp. 341-409.

Cuevas, E. \& A. Lugo. 1998. Dynamics of organic matter and nutrient return from litterfall in stands of ten tropical tree plantation species. Forest Ecology and Management 112: 263-279.

Del Arco, J. M., A. Escudero \& G. M. Vega. 1991. Effects of site characteristics on nitrogen retranslocation from senescing leaves. Ecology 72: 701-708.

Del Valle, J. I. 2003. Cantidad, calidad y nutrientes reciclados por la hojarasca fina en bosques pantanosos del Pacífico Sur Colombiano. Interciencia 28: 443-449.

Duivenvoorden, J. M. \& J. F. Lips. 1995. A land-ecologycal study of soils, vegetation and plant diversity in Colombian Amazonia. Tropenbos, Series 12. The Tropenbos Foundation. Wageningen.

Edwards, P. J. \& P. J. Grubb. 1982. Studies of mineral cycling in a montane rain forest in New Guinea IV. Soil characteristics and the division of mineral elements between the vegetation and soil. Journal of Ecology 70: 649-666.

Escudero, A. \& S. Mediavilla. 2003. Decline in photosynthetic nitrogen use efficiency with leaf age and nitrogen resorption as determinants of leaf life span. Journal of Ecology 91: 880-889.
Fassbender, H. W. \& V. Grimm. 1981. Ciclos biogeoquímicos en un ecosistema forestal de los Andes Occidentales de Venezuela II. Producción y descomposición de los residuos vegetales. Turrialba 31: 39-47.

Grimm, U. \& H. Fassbender. 1981. Ciclos biogeoquímicos en un ecosistema forestal de los Andes Occidentales de Venezuela: Ciclo hidrológico y translocación de elementos químicos con el agua. Turrialba 31: 89-99.

Grubb, P. J. 1989. The role of mineral nutrients in the tropics: a plant ecologist view. En: J. Proctor (ed.). Mineral nutrients in tropical forest and savanna ecosystems. Blackwell Scientific Publications. Boston. Pp. 417-440.

Grubb, P. J. \& P. J. Edwards. 1982. Studies of mineral cycling in a montane rain forest in New Guinea III. The distribution of mineral elements in the above-ground material. Journal of Ecology 70: 263-248.

Hagen-Thorn, A., I. Varnagiryte, B. Nihlgård \& K. Armolaitis. 2006. Autumn nutrient resorption and losses in four deciduous forest tree species. Forest Ecology and Management 228: 33-39.

Hirose, T. 1975. Relations between turnover rate, resource utility and structure of some plant populations: a study of the matter budgets. Journal of the Faculty of Science, University of Tokyo 11: 355-407.

Huang, J., X. Wang \& E. Yan. 2007. Leaf nutrient concentration, nutrient resorption and litter decomposition in an evergreen broad-leaved forest in eastern China. Forest Ecology and Management 239: 150-158.

Jaramillo, D. F. 1995. Andisoles del oriente antioqueño: caracterización química y fertilidad. Instituto de Ciencias Naturales y Ecología, Facultad de Ciencias, Universidad Nacional de Colombia. Medellín.

Jaramillo, D. F., M. T. Flórez \& L. N. Parra. 2006. Caracterización de un andisol de la cuenca de la quebrada Piedras Blancas, Oriente Antioqueño Colombiano. Suelos Ecuatoriales 36: 61-71. 
León, J. D. 2007. Contribución al conocimiento del ciclo de nutrientes en bosques montanos naturales de Quercus humboldtii y reforestados (Pinus patula y Cupressus lusitanica) de la región de Piedras Blancas, Antioquia (Colombia). Tesis doctoral. Universidad de Salamanca. Salamanca.

León, J. D., N. W. Osorio, J. A. Peláez-Silva \& L. H. Barreto. 2008. Estudio exploratorio de la actividad biológica del suelo en ecosistemas forestales altoandinos de Antioquia, Colombia. En: J. D. León (ed.). Ecología de bosques andinos: experiencias de investigación. Editorial La Carreta. Medellín. Pp. 149-180.

Londoño, A., D. C. Montoya, J. D. León \& M. I. González. 2005. Ciclaje y pérdida de nutrientes del suelo en bosques altoandinos de Antioquia, Colombia. Revista Facultad Nacional de Agronomía 60: 3717-3734.

Lundgren, B. 1978. Soil conditions and nutrient cycling under natural and plantation forests in Tanzanian highlands. Reports in Forest Ecology and Forest Soils 31. Swedish University of Agricultural Sciences. Uppsala. Pg. 428.

Malavolta, E. 1984. O Potasio e a Planta. Boletín Técnico 1. Asociação Brasileira para Pesquisa da Potassa e do Fosfato. Piracicaba. Pg. 61.

Martín, A., J. F. Gallardo \& I. Santa Regina. 1996. Aboveground litter production and bioelement potential return in an evergreen oak (Quercus rotundifolia) woodland near Salamanca (Spain). Annales des Sciences Forestières 53: 811-818.

Medina, E. 1984. Nutrient balance and physiological processes at the leaf level. En: E. Medina, H. A. Mooney \& C. Vázquez-Yañes (eds.). Physiological Ecology of Plants in the Wet Tropics. W. Junk. La Haya. Pp. 51-84.

Medina, E., E. Cuevas \& P. L. Weaver. 1981. Composición foliar y transpiración de especies leñosas del Pico del Este, Sierra de Luquillo, Puerto Rico. Acta Científica Venezolana 32: 159-165.
Medina, E., V. García \& E. Cuevas. 1990. Sclerophylly and oligotrophic environments: relationships between leaf structure, mineral nutrient content, and drought resistance in tropical rain forest of the upper Rio Negro region. Biotropica 22: 51-64.

Milla, R., P. Castro-Díez, M. Maestro-Martínez \& G. Montserrat-Martí. 2005. ¿Does the gradualness of leaf shedding govern nutrient resorption from senescing leaves in Mediterranean woody plants? Plant and Soil 278: 303-313.

O'Connell, A. M. \& K. V. Sankaran. 1997. Organic matter accretion, decomposition and mineralisation. En: E. K. Sadanandan \& A. G. Brown (eds.). Management of soil, nutrients and water in tropical plantation forests. Australian Centre for International Agricultural Research. Canberra. Pp. 443-480.

Newbery, D. M., I. J. Alexander \& J. A. Rother. 1997. Phosphorus dynamics in a lowland African rain forest: the influence of ectomycorrhizal trees. Ecological Monographs 67: 367-409.

Parker, G. G. 1983. Throughfall and stemflow in the forest nutrient cycle. Advances in Ecological Research 13: 57-133.

Proctor, J., J. M. Anderson, S. C. L. Fogden \& H. W. Vallack. 1983. Ecological studies in four contrasting lowland rainforest in Gunung Mulu National Park, Sarawak II. Litterfall, litter standing crop and preliminary observations on herbivory. Journal of Ecology 71: 261-283.

Proctor, J., C. Phillips, G. K. Duff, A. Heaney \& F. M. Robertson. 1989. Ecological studies on Gunung Silam, a small ultrabasic mountain in Sabah, Malaysia. Some forest processes. Journal of Ecology 77: 317-331.

Ramírez, J. A., C. M. Zapata, J. D. León \& M. I. González. 2007. Caída de hojarasca y retorno de nutrientes en bosques montanos andinos de Piedras Blancas, Antioquia, Colombia. Interciencia 32: 303-311.

Rodríguez, A. \& L. Molina. 1990. Cultivos múltiples, fertilización de maíz y fríjol en un andisol 
del oriente antioqueño. Suelos Ecuatoriales 20: 115-119.

Sánchez, P. A. 1976. Properties and management of soils in the tropics. Wiley, Nueva York. Pg. 618.

Santa Regina, I., S. Leonardi \& M. Rapp. 2001. Foliar nutrient dynamics and nutrient-use efficiency in Castanea sativa coppice stands of southern Europe. Forestry 74: 1-10.

Sanz, I. 1992. Estudio de la eficiencia en el uso del fósforo en la biomasa foliar de diversas especies leñosas de la cuenca del Duero. Trabajo de Licenciatura. Universidad de Salamanca. Salamanca.

Scott, D. A., J. Proctor \& J. Thompson. 1992. Ecological studies on a lowland evergreen rain forest on Maracá island, Roraima, Brazil II. Litter and nutrient cycling. Journal of Ecology 80: 705-717.

Silver, W. L. 1994. Is nutrient availability related to plant nutrient use in humid tropical dry forest? Oecologia 98: 336-346.

Smith, K., H. L. Gholz \& F. D. A. Oliveira. 1998. Litterfall and nitrogen-use efficiency of plantations and primary forest in the eastern Brazilian Amazon. Forest Ecology and Management 109: 209-220.

Steinhardt, U. 1979. Untersuchungen über den Wasser- und Nährstoffhaushalt eines andinen Wolkenwaldes in Venezuela. Göttinger Bodenkundliche Berichte 56: 1-185.

Tanner, E. V. J. 1977. Four montane rain forests of Jamaica: a quantitative characterization of the floristics, the soils, and the foliar mineral levels and a discussion of the interrelations. Journal of Ecology 65: 883-918.

Tschinkel, H. 1972. Factores limitantes del crecimiento de plantaciones de Cupressus lusitanica en Antioquia, Colombia. Revista Facultad Nacional de Agronomía 27: 3-55.
Tukey, H. B. Jr. 1970. The leaching of substances from plants. Annual Review of Plant Physiology 21: 305-324.

Van Heerwaarden, L. M., S. Toet \& R. Aerts. 2003. Current measures of nutrient resorption efficiency lead to a substantial underestimation of real resorption efficiency: facts and solutions. Oikos 101: 664-669.

Veneklaas, E. J. 1991. Litterfall and nutrient fluxes in two montane tropical rain forests, Colombia. Journal of Tropical Ecology 7: 319-336.

Vera, M., J. Cavelier \& J. Santamaría. 1999. Reabsorción de nitrógeno y fósforo foliar en árboles de bosques montanos en los Andes centrales de Colombia. Revista de Biología Tropical 47: 33-43.

Villela, D. \& J. Proctor. 1999. Litterfall mass, chemistry, and nutrient retranslocation in a monodominant forest on Maracá Island, Roraima, Brazil. Biotropica 31: 198-211.

Vitousek, P. M. 1982. Nutrient cycling and nutrient use efficiency. The American Naturalist 119: 553-572.

Vitousek, P. M. 1984. Litterfall, nutrient cycling, and nutrient limitation in tropical forests. Ecology 65: 285-298.

Vitousek, P. M. \& R. L. Sanford Jr. 1986. Nutrient cycling in moist tropical forest. Annual Review of Ecology and Systematics 17: 137167.

Vitousek, P. M., G. Gerrish, D. R. Turner, L. R. Walker \& D. Mueller-Dombois. 1995. Litterfall and nutrient cycling in four Hawaiian montane rainforests. Journal of Tropical Ecology 11: 189-203.

Weerakkody, J. \& D. Parkinson. 2006. Leaf litter decomposition in an upper montane rainforest in Sri Lanka. Pedobiologia 50: 387-395. 


\section{ANEXO FOTOGRÁFICO}

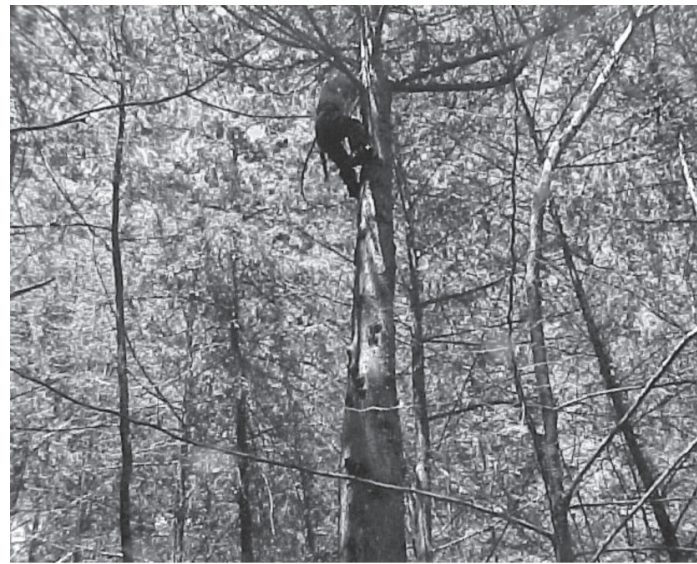

Foto 1

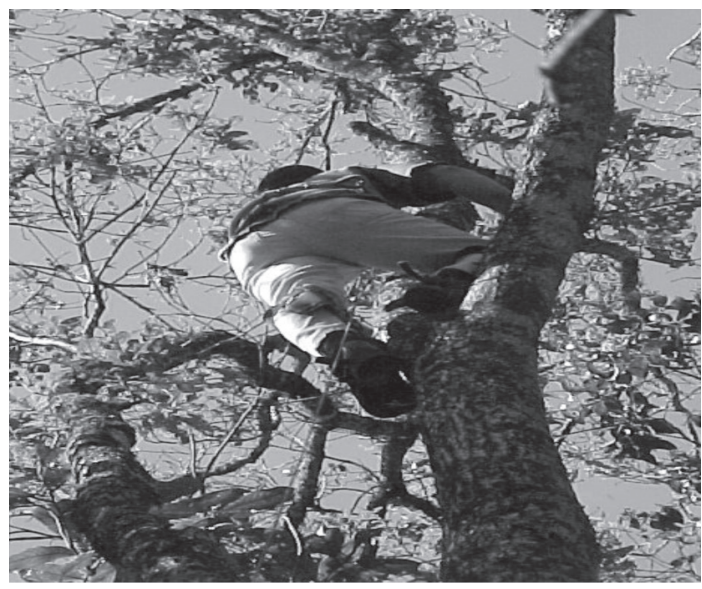

Foto 3

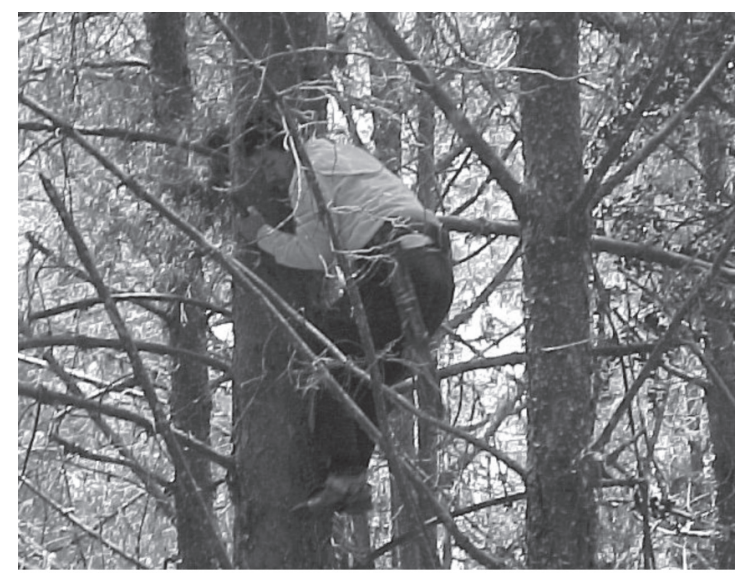

Foto 2

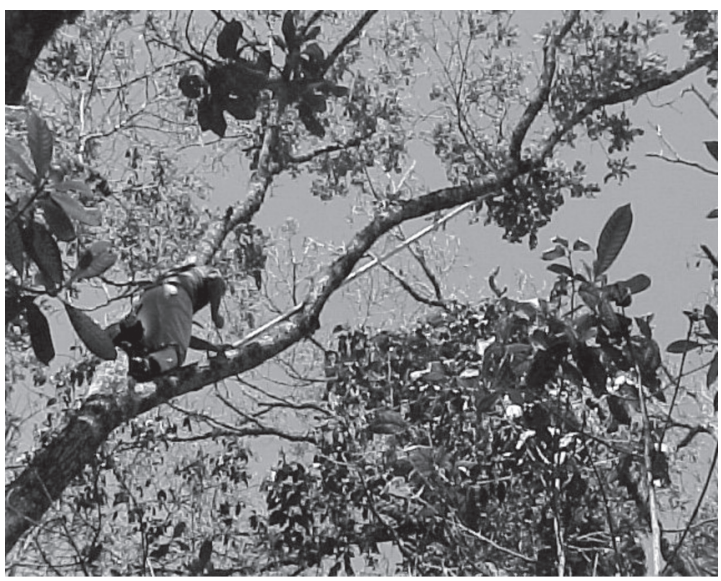

Foto 4

Fotos 1 y 2. Ascenso a ejemplares de pino para recolección de hojas.

Fotos 3 y 4. Ascenso a ejemplares de roble y empleo de tijera telescópica para recolección de hojas maduras. 


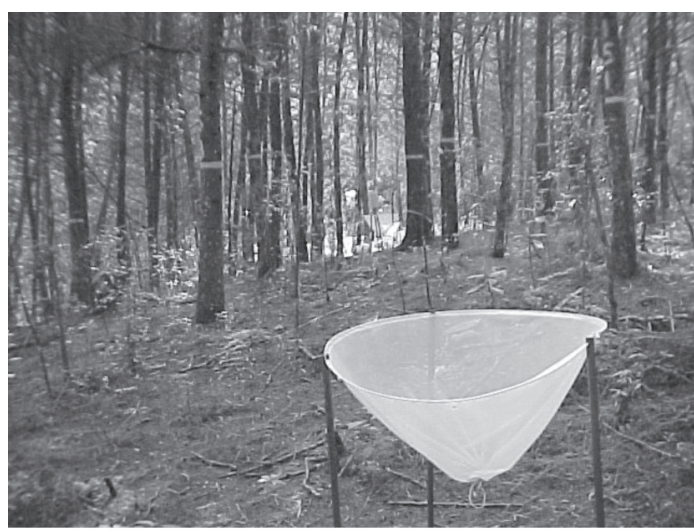

Foto 5

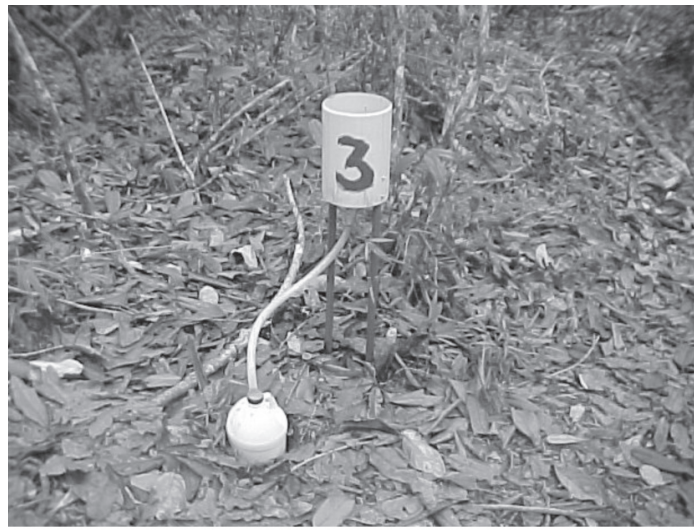

Foto 7

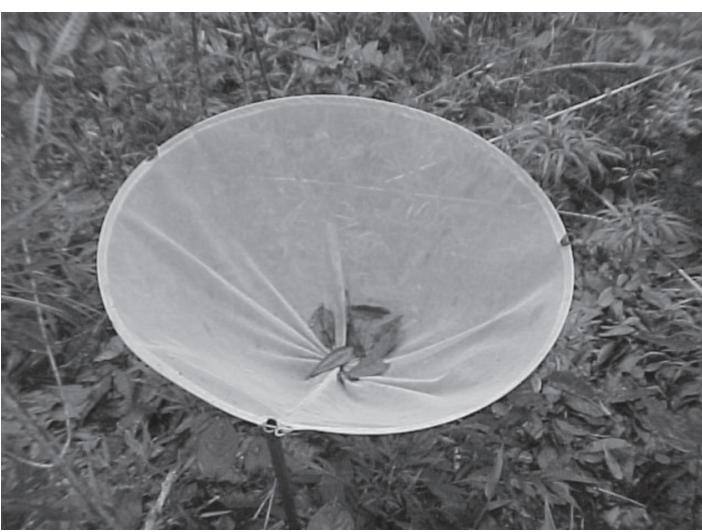

Foto 6

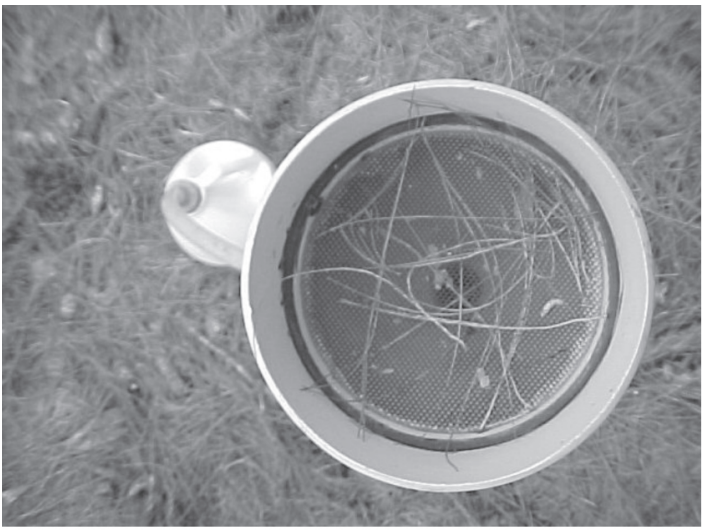

Foto 8

Fotos 5 y 6. Trampas de hojarasca fina.

Fotos 7 y 8. Pluviómetros dentro del bosque. 